\title{
UNCERTAINTY AND BUSINESS CYCLES: EXOGENOUS IMPULSE OR ENDOGENOUS RESPONSE?
}

\author{
Sydney C. Ludvigson \\ Sai Ma \\ Serena $\mathrm{Ng}$ \\ Working Paper 21803 \\ http://www.nber.org/papers/w21803 \\ NATIONAL BUREAU OF ECONOMIC RESEARCH \\ 1050 Massachusetts Avenue \\ Cambridge, MA 02138 \\ December 2015, Revised May 2018
}

The views expressed are those of the authors and do not necessarily reflect those of the Federal Reserve Board or the Federal Reserve System or the National Bureau of Economic Research.

NBER working papers are circulated for discussion and comment purposes. They have not been peer-reviewed or been subject to the review by the NBER Board of Directors that accompanies official NBER publications.

(C) 2015 by Sydney C. Ludvigson, Sai Ma, and Serena Ng. All rights reserved. Short sections of text, not to exceed two paragraphs, may be quoted without explicit permission provided that full credit, including $\odot$ notice, is given to the source. 
Uncertainty and Business Cycles: Exogenous Impulse or Endogenous Response?

Sydney C. Ludvigson, Sai Ma, and Serena Ng

NBER Working Paper No. 21803

December 2015, Revised May 2018

JEL No. E00,E32,E44,G01,G12

\section{ABSTRACT}

Uncertainty about the future rises in recessions. But is uncertainty a source of business cycles or an endogenous response to them, and does the type of uncertainty matter? We propose a novel SVAR identification strategy to address these questions via inequality constraints on the structural shocks. We find that sharply higher macroeconomic uncertainty in recessions is often an endogenous response to output shocks, while uncertainty about financial markets is a likely source of output fluctuations. But the findings also suggest that macroeconomic uncertainty plays an important role in recessions, by substantially amplifying downturns caused by other shocks.

Sydney C. Ludvigson

Department of Economics

New York University

19 W. 4th Street, 6th Floor

New York, NY 10002

and NBER

sydney.ludvigson@nyu.edu

Sai Ma

Federal Reserve Board

C Ave \& 20th Street NW

Washington, DC 20551

sai.ma@frb.gov

\author{
Serena $\mathrm{Ng}$ \\ Department of Economics \\ Columbia University \\ 420 West 118th Street \\ New York, NY 10027 \\ and NBER \\ Serena.Ng@columbia.edu
}




\section{Introduction}

A large literature in macroeconomics investigates the relationship between uncertainty and business cycle fluctuations. Interest in this topic has been spurred by a growing body of evidence that uncertainty rises sharply in recessions. This evidence is robust to the use of specific proxy variables such as stock market volatility and forecast dispersion as in Bloom (2009), or a broad-based measure of macroeconomic uncertainty, as in Jurado, Ludvigson, and $\mathrm{Ng}$ (2015) (JLN hereafter). But while this evidence substantiates a role for uncertainty in deep recessions, the question of whether uncertainty is an exogenous source of business cycle fluctuations or an endogenous response to economic fundamentals is not fully understood. Existing results are based on convenient but restrictive identifying assumptions and have no explicit role for financial markets, even though uncertainty measures are strongly correlated with financial market variables. This paper considers a novel identification strategy to disentangle the causes and consequences of real and financial uncertainty.

The question of causality and the identification of exogenous variation in uncertainty is a long-standing challenge of the uncertainty literature. The challenge arises in part because there is no single uncertainty model, hence no theoretical consensus on whether the uncertainty that accompanies deep recessions is primarily a cause or effect (or both) of declines in economic activity. In fact, theory is even ambiguous about the sign of the effect, as we discuss below.

A separate challenge of the uncertainty literature pertains to the origins of uncertainty. Classic theories assert that uncertainty originates from economic fundamentals such as productivity, and that such real economic uncertainty, when interacted with market frictions, discourages real activity. But some researchers have argued that uncertainty dampens the economy through its influence on financial markets (e.g., Gilchrist, Sim, and Zakrajsek (2010)) or through sources of uncertainty specific to financial markets (e.g., Bollerslev, Tauchen, and Zhou (2009)). Moreover, as surveyed by Ng and Wright (2013), all the post-1982 recessions have origins in financial markets, and these recessions have markedly different features from recessions where financial markets play a passive role. From this perspective, if financial shocks are subject to time-varying volatility, financial market uncertainty-as distinct from real economic uncertainty-could be a key player in recessions, both as a cause and as a propagating mechanism. Yet so far the literature has not disentangled the contributions of real versus financial uncertainty to business cycle fluctuations.

Econometric analyses aimed at understanding the role of uncertainty for business cycle fluctuations face their own challenges. Attempts to identify the "effects" of uncertainty shocks in existing empirical work are primarily based on recursive schemes within the framework of vector-autoregressions (VAR). ${ }^{1}$ While a recursive structure is a convenient starting point, it is

\footnotetext{
${ }^{1}$ See Bachmann, Elstner, and Sims (2013), Bloom (2009), Bloom (2014), Bekaert, Hoerova, and Duca (2013),
} 
ultimately unsatisfactory as an identification strategy for a study on uncertainty and business cycles. Not only do the existing studies differ according to whether uncertainty is ordered ahead of or after real activity variables in the VAR, there is no compelling theoretical reason to restrict the timing of the relationship between uncertainty (a second moment variable) and real activity (a first moment variable). Uncertainty could comove contemporaneously with real activity both because it is an exogenous impulse driving business cycles and because it responds endogenously to first moment shocks. Recursive structures explicitly rule this out, since they presume that some variables respond only with a lag to others. Other commonly used VAR identification strategies, such as sign restrictions, long-run restrictions, and instrumental variables estimation, are likewise problematic, as we discuss further below.

It is with these challenges in mind that we return to the questions posed above: is uncertainty primarily a source of business cycle fluctuations or a consequence of them? And what is the relation of real versus financial uncertainty to business cycle fluctuations? The objective of this paper is to establish a set of stylized facts that addresses these questions econometrically. To do so, we take a two-pronged approach. First, we explicitly distinguish macro uncertainty $U_{M t}$, from financial uncertainty $U_{F t}$ and force their effects to be orthogonal. These data are included together in a structural vector autoregression (SVAR) along with a measure of real activity $Y_{t}$ to evaluate their possibly distinct roles in business cycle fluctuations. Second, we propose a novel identification strategy that allows for simultaneous feedback between uncertainty and real activity using what we shall refer to as shock-based restrictions. Whereas SVARs are typically identified using a priori restrictions on the structural parameters, we acheive identification by using inequality constraints on the structural shocks to reinforce and interpret what the data already suggest.

More precisely, we impose two sets of shock-based restrictions. The first is a set of "event constraints" that require the identified shocks to have defensible properties during special episodes of history for which a broad historical understanding of the events of the time would suggest a certain behavior of the structural shocks. We augment this understanding by using the data itself, i.e., the set of solutions consistent only with the standard reduced-form covariance restrictions, to locate distinguishing characteristics of the two different types of uncertainty shocks. The second is a set of "external variable constraints" that require the identified uncertainty shocks to exhibit a non-zero absolute correlation with certain variables external to the VAR that should be informative about uncertainty shocks. While our shock-based restrictions do not in general permit point identification, the moment inequalities generated by these constraints, along with the reduced-form covariance restrictions, yield identified sets that paint a fairly clear picture regarding the role of financial and macro uncertainty during recessions.

The empirical exercise additionally requires that appropriate measures of macro and finanGilchrist, Sim, and Zakrajsek (2010), and JLN. 
cial uncertainty be available. Our measures of uncertainty quantify the magnitude of unpredictability about the future. As in JLN, the macro uncertainty index $U_{M t}$ measures a common component in the time-varying volatilities of $h$-step ahead forecast errors across a large number of macroeconomic series that include variables from three categories: real activity (the most numerous), price, and financial. The same approach is used here to construct a broad-based index of financial uncertainty $U_{F t}$ that is based solely on financial market data and has never been used in the literature. We also study the Baker, Bloom, and Davis (2016) economic policy uncertainty (EPU) index, an alternative to the JLN macro uncertainty measure that is arguably relevant for real activity based macro uncertainty.

Our main results may be stated as follows. First, positive shocks to financial uncertainty are found to cause a sharp and persistent decline in real activity, lending support to the hypothesis that heightened uncertainty is an exogenous impulse that causes recessions. In contrast to preexisting empirical work that uses recursive identification schemes (e.g., Bloom (2009), JLN), we trace the source of this result specifically to financial market uncertainty. However there is little evidence that negative shocks to real activity have adverse effects on financial uncertainty.

Second, the results suggest that sharply higher macro and policy uncertainty in recessions is best characterized as an endogenous response to business cycle fluctuations. That is, negative economic activity shocks are found to cause increases in both macro and policy uncertainty, but there is less evidence that positive shocks to macro or policy uncertainty cause lower economic activity. Indeed, in most estimations the opposite is true: exogenous shocks to macro and policy uncertainty are found to initially increase real activity, consistent with "growth options" theories discussed below.

Third, the results indicate that variation in macro uncertainty is likely to be an important force for amplifying recessions even if it doesn't cause them. An analysis that hypothetically restricts to zero the endogenous responses of macro uncertainty to adverse shocks elsewhere in the system (in contrast to our estimates), points to a substantial role for macro uncertainty in magnifying and propagating the effects of other shocks.

Fourth, an inspection of our identified solution sets shows that the admissible SVARs reflect a non-zero contemporaneous correlation between $U_{F t}$ and $Y_{t}$, as well as between $U_{M t}$ and $Y_{t}$, something that is inconsistent with any recursive ordering. Tests of the validity of a recursive structure are easily rejected by the data.

Finally, all three estimated shocks systematically exhibit strong non-Gaussian features such as skewness and excess kurtosis. This is of interest because structural economic modeling and most Bayesian estimation techniques typically assume Gaussianity.

Looking across configuration of variables and restrictions considered, the finding that most stands out is the strong repercussions for real activity of financial uncertainty shocks. And, while we find little evidence that macro uncertainty is a cause of lower economic growth as 
hypothesized by many theoretical models, our results suggest that macro uncertainty plays a crucial role in amplifying the contractionary effects of other adverse shocks. In other words, uncertainty shocks are not all alike. Distinguishing between the two types of uncertainty thus appears necessary for understanding the roles that uncertainty plays in economic fluctuations.

The rest of this paper is organized as follows. Section 2 reviews related literature. Section 3 discusses the econometric framework and identifying assumptions, and compares our approach to other methodologies. Section 4 discusses the data and Section 5 the implementation. Section 6 presents the main results. Section 7 reports estimations of several additional cases and extensions. Section 8 summarizes and concludes. A number of additional results and information are reported in the Online Appendix. Shock-based restrictions are likely to have promise in other applications. A paper with greater detail on the methodology proposed here with additional applications can be found in Ludvigson, Ma, and $\mathrm{Ng}$ (2016).

\section{Related Literature}

A large literature addresses the question of uncertainty and its relation to economic activity. $^{2}$ Besides the evidence cited above for the U.S., Nakamura, Sergeyev, and Steinsson (2017) estimate growth rate and volatility shocks for 16 developed countries and find that they are substantially negatively correlated. Theories for which uncertainty plays a key role differ widely on the question of whether this correlation implies that uncertainty is primarily a cause or a consequence of declines in economic activity.

One strand of the literature proposes uncertainty as a cause of lower economic growth. This includes models of the real options effects of uncertainty (Bernanke (1983), McDonald and Siegel (1986)), models in which uncertainty influences financing constraints (Gilchrist, Sim, and Zakrajsek (2010), Arellano, Bai, and Kehoe (2011)), or precautionary saving (Basu and Bundick (2017), Leduc and Liu (2016), Fernández-Villaverde, Pablo Guerrón-Quintana, and Uribe (2011)). These theories almost always presume that uncertainty is an exogenous shock to the volatility of some economic fundamental. Some theories presume that higher uncertainty originates directly in the process governing technological innovation, which subsequently causes a decline in real activity (e.g., Bloom (2009), Bloom, Floetotto, Jaimovich, Saporta-Eksten, and Terry (2018)). According to these theories, positive macro uncertainty shocks should cause declines in real economic activity. But while this theoretical literature has focused on uncertainty originating in economic fundamentals, the empirical literature has typically evaluated those theories using uncertainty proxies that are strongly correlated with financial market variables. This practice raises the question of whether it is real economic uncertainty or financial market uncertainty (or both) that is the driver of recessions, a question of interest to our

\footnotetext{
${ }^{2}$ This literature has become voluminous. See Bloom (2014) for a recent review of the literature.
} 
investigation.

A second strand of the literature postulates that higher macro uncertainty arises solely as a response to lower economic growth. In these theories there is no exogenous uncertainty shock at all and all uncertainty variation is endogenous. Some theories presume that bad times incentivize risky behavior (Bachmann and Moscarini (2011), Fostel and Geanakoplos (2012)), or reduce information and with it the forecastability of future outcomes (Van Nieuwerburgh and Veldkamp (2006), Fajgelbaum, Schaal, and Taschereau-Dumouchel (2017), Ilut and Saijo (2016)), or provoke new and unfamiliar economic policies with uncertain effects (Pástor and Veronesi (2013)), or create a greater misallocation of capital across sectors ( $\mathrm{Ai}, \mathrm{Li}$, and Yang (2015)), or generate endogenous countercyclical uncertainty in consumption growth because investment is costly to reverse (Gomes and Schmid (2016)).

And yet a third literature has raised the possibility that some forms of uncertainty can actually increase economic activity. "Growth options" theories of uncertainty postulate that a mean-preserving spread in risk generated from an unbounded upside coupled with a limited downside can cause firms to invest and hire, since the increase in mean-preserving risk increases expected profits. Such theories were often used to explain the dot-com boom. Examples originate in early work by Oi (1961), Hartman (1972), and Abel (1983), and more recently Bar-Ilan and Strange (1996), Pastor and Veronesi (2006), Kraft, Schwartz, and Weiss (2018), Segal, Shaliastovich, and Yaron (2015).

As this brief literature review makes plain, there is no single uncertainty theory or allencompassing structural model that we can use to link with data. Put simply, the body of theoretical work does not provide precise identifying restrictions for empirical work. Instead, what the literature presents is a wide range of theoretical predictions about the relationship between uncertainty and real economic activity that are also ambiguous about the sign of the relationship. The absence of a theoretical consensus on this relationship, along with the sheer number of theories and limited body of evidence on the structural elements of specific models, underscores the extent to which the question of cause and effect is fundamentally an empirical one.

Of course, all empirical studies of this nature require identifying assumptions. But commonly used SVAR identification schemes appear ill equipped to address the empirical questions at hand. Recursive identification schemes are inappropriate because, by construction, they rule out the possibility that uncertainty and real activity could influence one another within the period. Sign restrictions on impulse responses are inappropriate, since theory is ambiguous about the sign of the relationship. Zero-frequency restrictions are difficult to motivate as the long-run effects of uncertainty shocks have not been theorized. Instrumental variable analysis is challenging, since instruments that are credibly exogenous are difficult if not impossible to find for this application. All of these considerations motivate the alternative identification strategy 
proposed in this paper.

\section{Econometric Framework}

We consider a baseline system with $n=3$ variables: $\mathbf{X}_{t}=\left(U_{M t}, Y_{t}, U_{F t}\right)^{\prime}$, where $U_{M t}$ denotes the JLN index of macro uncertainty, $Y_{t}$ denotes a measure of real activity, and $U_{F t}$ denotes the index of financial uncertainty. We suppose that $\mathbf{X}_{t}$ has a reduced-form finite-order autoregressive representation $\mathbf{X}_{t}=\sum_{j=1}^{p} \mathbf{A}_{j} \mathbf{X}_{t-j}+\boldsymbol{\eta}_{t}, \boldsymbol{\eta}_{t} \sim(0, \boldsymbol{\Omega}), \quad \boldsymbol{\Omega}=\mathbf{P} \mathbf{P}^{\prime}$ where $\mathbf{P}$ is the unique lowertriangular Cholesky factor with non-negative diagonal elements. The reduced form parameters are collected into $\boldsymbol{\phi}=\left(\operatorname{vec}\left(\mathbf{A}_{1}\right)^{\prime} \ldots \operatorname{vec}\left(\mathbf{A}_{p}\right)^{\prime}, \quad \operatorname{vech}(\boldsymbol{\Omega})^{\prime}\right)^{\prime}$. The reduced form innovations $\boldsymbol{\eta}_{t}=\left(\eta_{M t}, \eta_{Y t}, \eta_{F t}\right)^{\prime}$ are related to the structural shocks $\mathbf{e}_{t}=\left(e_{M t}, e_{Y t}, e_{F t}\right)^{\prime}$ by an invertible matrix $\mathbf{H}$ :

$$
\boldsymbol{\eta}_{t}=\mathbf{H} \boldsymbol{\Sigma} \mathbf{e}_{t} \equiv \mathbf{B e}_{t}, \quad \mathbf{e}_{t} \sim\left(0, \mathbf{I}_{\mathbf{K}}\right), \quad \operatorname{diag}(\mathbf{H})=1,
$$

where $\mathbf{B} \equiv \mathbf{H} \boldsymbol{\Sigma}$, and $\boldsymbol{\Sigma}$ is a diagonal matrix with variance of the shocks in the diagonal entries. The structural shocks $\mathbf{e}_{t}$ are mean zero with unit variance, serially and mutually uncorrelated. We adopt the unit effect normalization that $H_{j j}=1$ for all $j$.

The goal of the exercise is analyze the dynamic effects of $\mathbf{e}_{t}$ on $\mathbf{X}_{t}$. Let "hats" denote estimated variables. Since the autoregressive parameters $\mathbf{A}_{j}$ can be consistently estimated under regularity conditions, the sample residuals $\hat{\boldsymbol{\eta}}_{t}(\hat{\boldsymbol{\phi}})$ are consistent estimates of $\boldsymbol{\eta}_{t}$. The empirical SVAR problem reduces to finding $\mathbf{B}$ from $\hat{\boldsymbol{\phi}}$. But there are 9 parameters in $\mathbf{B}$ and the covariance structure of $\hat{\boldsymbol{\eta}}_{t}$ only provides $n(n+1) / 2=6$ restrictions in the form

$$
\bar{g}_{Z}(\mathbf{B})=\operatorname{vech}(\hat{\mathbf{\Omega}})-\operatorname{vech}\left(\mathbf{B B}^{\prime}\right)=\mathbf{0}
$$

Thus the model is under-identified as there can be infinitely many solutions satisfying the covariance restrictions $\bar{g}_{Z}(\mathbf{B})=\mathbf{0}$. Let these uncountably many solutions be collected into the set $\hat{\mathcal{B}}=\left\{\mathbf{B}=\hat{\mathbf{P}} \mathbf{Q}: \mathbf{Q} \in \mathbb{O}_{n}, \operatorname{diag}(\mathbf{B}) \geq 0, \bar{g}_{Z}(\mathbf{B})=\mathbf{0}\right\}$, where $\mathbb{O}_{n}$ is the set of $n \times n$ orthonormal matrices. We shall refer to $\hat{\mathcal{B}}$ as the unconstrained set for short, with the understanding that it is not completely unconstrained given the imposition of the reduced-form covariance restrictions. To simply notation, the dependence of $\hat{\mathcal{B}}$ on $\mathbf{Q}$ and $\hat{\phi}$ is suppressed.

Point identification requires restrictions beyond the ones implied by the covariance structure to reduce $\hat{\mathcal{B}}$ to a singleton. As discussed above, the theories reviewed in previous section do not lend support to identification schemes commonly used in the literature. One possibility is to turn to external variables. An example relevant to our work is Stock and Watson (2012), in which either stock market volatility or the EPU index of Baker, Bloom, and Davis (2016) are used as external instrumental variables (IV) to identify the effects of uncertainty shocks. Our analysis differs not only because we have two types of uncertainty and are interested in the dynamic effects of all shocks in the model. The main difference is that our procedure 
explicitly recognizes that macro uncertainty, policy uncertainty, and financial uncertainty are all endogenous variables. As such, valid instruments, which must be exogenous, are hard to find.

We address these challenges by putting restrictions on the behavior of the structural shocks. Although the unconstrained set can be uncountably large, our proposed identifying restrictions explicitly recognize that not every solution in $\hat{\mathcal{B}}$ is equally credible. We refer to these as shockbased restrictions since we use features of the shocks to decide if a solution in the unconstrained set should be kept. Even though the stated goal of any SVAR exercise is to identify $\mathbf{e}_{t}$, it is somewhat surprising that little attention is paid to the shocks themselves. Before turning to the restrictions, we first discuss some facts that motivate them.

\subsection{Motivating Facts}

Since we are interested in understanding the possible role of different types of uncertainty in economic fluctuations, it is useful to isolate episodes of heightened financial and macro uncertainty. Consider first financial uncertainty. In the sample we study, the two largest financial uncertainty episodes are the 1987 stock market crash, and the Great Financial Crisis/Great Recession of 2008-2009.

On Monday October 19, 1987, the Dow Jones Industrial Average dropped 22.6 percent, the largest one-day stock market decline in history. Popular explanations include the rapidly rising globalization of financial markets and financial innovations associated with index futures and portfolio insurance. A belief that such financial innovations played a predominate role in the crash was sufficiently widespread that new regulations for exchange trading, such as "circuit breakers," and an overhaul of trade clearing protocols were developed in the aftermath. ${ }^{3}$

In October of 2008, the Dow Jones Industrial average began a pronounced decline and subsequently fell more than $50 \%$ over a period of 17 months. The collapse in the market over this period has been associated with a broad-based Great Financial Crisis (GFC) that is often cited as a "trigger" of the Great Recession (GR). ${ }^{4}$ Many possible contributors to the crisis have been noted, including problems with subprime lending and a preceding housing boom. But at least some of the variation in financial uncertainty appears to have its origins in securities markets. Financial intermediaries played a large role in the crisis, primarily because they hold vast portfolios of financial securities. Speculative trading activities by large financial institutions such as AIG, Lehman Brothers, and Bear Stearns, possibly spurred by a mistaken pricing of risk, have been placed at the center of the crisis by some analyses (e.g., Glaeser, Santos, and Weyl (2017)). Several highly leveraged financial institutions (BNP Paribas, Northern Rock) experienced a total collapse in liquidity that began August of 2007, preceding the recession. And

\footnotetext{
${ }^{3}$ See for example, https://www.federalreservehistory.org/essays/stock_market_crash_of_1987

${ }^{4}$ https://en.wikipedia.org/wiki/Financial_crisis_of_2007-2008
} 
uncertainty about the value of new products of financial innovation have been cited as pertinent to the financial crisis, including the securitization of mortgages and other debt obligations, and the rapid growth in credit default swaps. ${ }^{5}$ In short, a defining feature of both the 1987 crash and the Great Financial Crisis is that factors originating specifically in financial markets appeared to play an important role in heightened financial uncertainty.

With this in mind, we ask what can be said about the uncertainty shocks themselves before imposing any identifying restrictions. To address this question, we construct the unconstrained set $\hat{\mathcal{B}}$, which is based on the reduced-form covariance restrictions alone, and then study when big shocks in this set have occurred over the course of fifty years in our sample, by searching across the unconstrained set $\hat{\mathcal{B}}$ for the month in which the uncertainty shocks $e_{F t}(\mathbf{B})$ and $e_{M t}(\mathbf{B})$ are largest.

To construct the unconstrained solution set $\hat{\mathcal{B}}$, we initialize $\mathbf{B}$ to be the unique lowertriangular Cholesky factor of $\hat{\mathbf{\Omega}}$ with non-negative diagonal elements, $\hat{\mathbf{P}}$, and then rotate it by $K=1.5$ million random orthogonal matrices Q. Each rotation begins by drawing an $n \times n$ matrix $\mathbf{M}$ of $\operatorname{NID}(0,1)$ random variables. Then $\mathbf{Q}$ is taken to be the orthonormal matrix in the $\mathbf{Q R}$ decomposition of $\mathbf{M}$. Since $\mathbf{B}=\hat{\mathbf{P}} \mathbf{Q}$, the procedure imposes the covariance restrictions $\operatorname{vech}(\boldsymbol{\Omega})=\operatorname{vech}\left(\mathbf{B B}^{\prime}\right)$ by construction. Let $\mathbf{e}_{t}(\mathbf{B})=\mathbf{B}^{-1} \hat{\boldsymbol{\eta}}_{t}$ be the shocks implied by a $\mathbf{B} \in \hat{\mathcal{B}}$ for given $\hat{\boldsymbol{\eta}}_{t}$. The moments implied by the covariance structure alone give us 1.5 million values of $\mathbf{B}$, and thus 1.5 million unconstrained values of $\mathbf{e}_{t}(\mathbf{B})$ for $t=1, \ldots T$.

Searching over the 1.5 million rotations, we find that the date in our sample with the most maxima in $e_{F t}(\mathbf{B})$ (31\% of them) is 2008:09, which happens to be the month when Lehman filed for bankruptcy and when the stock market fell nearly 800 points. The date with the second most maxima in $e_{F t}(\mathbf{B})$ (25\% of them) is 1987:10, the month of the stock market crash. Looking at the 1.5 million values of $e_{F t}(\mathbf{B})$ obtained for 2008:09, we find that its distribution is right skewed with few negative values: $30 \%$ of the $e_{F t}(\mathbf{B})$ in 2008:09 have a value of 4 (standard deviations) or larger, $20 \%$ have a value of 5 or larger, while $5 \%$ of them take on a value of 6 or larger. For the 1.5 million values of $e_{F t}(\mathbf{B})$ obtained in 1987:10, we find similarly that $25 \%$ of the $e_{F t}(\mathbf{B})$ in 1987:10 have a value of four (standard deviations) or larger, while $5 \%$ of them take on a value of 5 or larger. Thus, the covariance structure of the data alone provides overwhelming evidence of a large positive financial uncertainty shock in the months of the 1987 crash and the 2008 Lehman collapse.

We repeat this exercise for the macro uncertainty shocks. The date with the most maxima in $e_{M t}(\mathbf{B})$ across all 1.5 million rotations in $\hat{\mathcal{B}}$ (32\% of them) is again 2008:09, the month of the Lehman collapse, while the date with the second most maxima is 1970:12. Looking at the 1.5 million values of $e_{M t}(\mathbf{B})$ obtained for 2008:09, we find a distribution that is very close to that of $e_{F t}(\mathbf{B}): 33 \%$ have a value of 4 or larger, $20 \%$ have a value of 5 or larger, and $5 \%$ have

\footnotetext{
5 "FT Martin Wolf - Reform of Regulation and Incentives". Financial Times. June 23, 2009.
} 
a value of 6 or larger. For the 1970:12 date, we find that $25 \%$ of the 1.5 million values of $e_{M t}(\mathbf{B})$ have a value of 4 or larger, while $10 \%$ of them take on a value of 5 or larger. Thus, the covariance structure of the data alone provides overwhelming evidence of a large positive macro uncertainty shock in the month of the 2008 Lehman collapse and in December of 1970.

What might be the significance of the last month in 1970 for macro uncertainty? Unlike 2008:09, this date is not associated with a single salient event such as the Lehman collapse. But further inspection shows that the big macro uncertainty shocks found in 1970:12 have their origins in uncertainty about real economic activity. ${ }^{6}$ The late 1960s and early 1970 s were a time of rising anxieties on a number of fronts given the twin wars in Vietnam and Cambodia and accelerating inflation. But, arguably, the single most relevant source of real economic uncertainty at the end of 1970 involved the likelihood and consequences of a collapse of the Bretton Woods system. By December of 1970, it was evident that the large U.S. balance of payments deficit of the time was incompatible with the gold standard, an acknowledgement that prompted periodic runs on the dollar and growing unease over the possible policy response. By the time of the so-called Nixon "shock" in August of 1971, a little over six months after our identified big real uncertainty shock, it was widely recognized that the $\$ 35$-per-ounce of gold conversation rate was unsustainable, and that the collapse of Bretton Woods was inevitable. Thus a historical reading of the times would place the period of genuine uncertainty surrounding the collapse of Bretton Woods sometime earlier than August 1971, when there was still room for reasoned speculation. From an inspection of the unconstrained set, it is apparent that the date with the biggest shock in the twelve month run-up to the abandonment of the gold standard is 1970:12. Evidently, this date represents a big shock episode for the real activity component of $U_{M t}$, one that appears to be relatively unimportant for financial uncertainty. Conversely, the month of the 1987 stock market crash represents a big shock episode for $U_{F t}$, one that is relatively unimportant for macro/real uncertainty. We use these facts below to help discern the possibly distinct roles of macro and financial uncertainty for real activity.

We argue that other major economic events in our sample, even if they are not big shock events in $\hat{\mathcal{B}}$, still justify weaker restrictions on the signs of the uncertainty shocks during specific months. On October 6, 1979, Paul Volcker announced the results of an unscheduled FOMC meeting in which it was decided that a new nonborrowed reserves instrument approach to the implementation of monetary policy would supersede the federal funds rate instrument approach used for years prior, in an effort to bring down accelerating inflation. In July/August of 2011, the U.S. House of Representatives broke with long-standing precedent to demand that the U.S. President negotiate over deficit reduction in exchange for an increase in the debt ceiling. We

\footnotetext{
${ }^{6}$ When we replace $U_{M t}$ with a sub-index based only on the real activity variables in the macro index, we find that 1970:12 is the date with the greatest number of maxima in the shocks to real activity uncertainty. We investigate this sub-index further below.
} 
argue that a credible identification scheme would not identify October 1979 or the months associated with the 2011 debt ceiling crisis as periods of below average uncertainty shocks. Similarly, a credible identification scheme would not identify the Great Recession-the most protracted economic downturn of the post-war period-as an episode in which real activity shocks were above average.

Our restrictions also employ variables external to the SVAR to help identify uncertainty shocks. What type of external variables are likely to encode information about uncertainty shocks? We argue that both the stock market and price of safe-haven assets such as gold are two such variables. Empirically, it has long been understood that periods of high stock market volatility coincide with movements downward in the aggregate stock market. ${ }^{7}$ The correlation between the CBOE Volatility Index (VIX) and the log excess stock market return on the CRSP value-weighted stock market index is -0.40 from January 1990 (when the standard VIX series begins) to the end of our sample. This correlation is also relevant for $U_{F t}$, since its behavior is dominated by the volatility of equity returns. ${ }^{8}$ Such a negative correlation between uncertainty shocks and the ex-post stock return is implied by a large body of extant macro and finance theories. For example, the Capital Asset Pricing Model (CAPM) of Sharpe (1964) and Lintner (1965) implies a perfect negative correlation since the stock market risk premium (which moves inversely to the ex-post return) is perfectly correlated with shocks to financial uncertainty. More recent asset pricing theories that emphasize the importance of uncertainty shocks, such as those in Bollerslev, Tauchen, and Zhou (2009) and Campbell, Giglio, Polk, and Turley (2018), argue that the volatility of volatility in financial markets creates an additional source of negative correlation between uncertainty shocks and ex-post stock market returns. Likewise, the value of gold is likely to contain information about uncertainty shocks, including macroeconomic uncertainty with origins outside of equity markets. Examples of these forms of uncertainty are natural disasters, terrorist attacks, political coups and revolutions, as considered in Baker and Bloom (2013), but also unpredictable inflation, interest rates, or energy prices. Our premise is that plausibly exogenous increases in uncertainty of this nature have typically been associated with increases in returns on quintessential safe-haven assets, the archetypal example being gold. ${ }^{9}$

\footnotetext{
${ }^{7}$ See, for example, French, Schwert, and Stambaugh (1987); Schwert (1989); Nelson (1991); Campbell and Hentschel (1992); Engle and Ng (1993), and Whaley (2000).

${ }^{8}$ The correlation between the VIX and $U_{F t}$ is 0.85 in the sample that starts in 1990. The correlation between the VXO volatility index (which goes back to the beginning of our sample) and $U_{F t}$ is 0.75 .

${ }^{9}$ Piffer and Podstawski (2017) suggest using variation in the real price of gold around specific events as an instrumental variable to identify uncertainty shocks. We instead use it as an informative external variable for macro uncertainty shocks, without requiring it to be a valid instrument.
} 


\subsection{Shock-Based Constraints}

Motivated by the historical facts just discussed, we now consider two types of shock-based restrictions to help with identification: event constraints, and external variables constraints.

A. Event Constraints Event constraints are unusual episodes of history in which a broadbased (historical and statistical) reading of the times would suggest a specific feature of the structural shocks. The idea is that a credible identification scheme should produce shocks that are not grossly at variance with our ex-post understanding of events, at least during periods of special interest. Specifically, we require that any $\mathbf{e}_{t}(\mathbf{B})$ formed from $\mathbf{B} \in \hat{\mathcal{B}}$ satisfy the following event constraints:

$$
\begin{aligned}
& \text { i } \bar{g}_{E 1}: e_{F \bar{\tau}_{1}} \geq \bar{k}_{1} \text { at } \bar{\tau}_{1}=1987: 10 \\
& \text { ii } \bar{g}_{E 2}:\left(e_{F \bar{\tau}_{2}} \geq \bar{k}_{2}\right) \vee\left(e_{M \bar{\tau}_{2}} \geq \bar{k}_{3}\right) \text { at } \bar{\tau}_{2}=2008: 09 \\
& \text { iii } \bar{g}_{E 3}: e_{M \bar{\tau}_{3}} \geq \bar{k}_{4} \text { at } \bar{\tau}_{3}=1970: 12 \text {. } \\
& \text { iv } \bar{g}_{E 4}: 0 \geq \sum_{t=\bar{\tau}_{3}} e_{Y t} \text { for } \bar{\tau}_{4} \in[2007: 12,2009: 06] \\
& \text { v } \bar{g}_{E 5}: e_{M \bar{\tau}_{4}} \geq 0 \text { and } e_{F \bar{\tau}_{5}} \geq 0 \text { at } \bar{\tau}_{5}=1979: 10 \\
& \text { vi } \bar{g}_{E 6}: e_{M \bar{\tau}_{5}} \geq 0 \text { and } e_{F \bar{\tau}_{6}} \geq 0 \text { at } \bar{\tau}_{6} \in[2011: 07,2011: 08]
\end{aligned}
$$

The first three constraints pertain to the big shock events discussed above. Condition (i) requires that the financial uncertainty shock found in period $\bar{\tau}_{1}$ of October 1987 (black Monday) be large and exceed $\bar{k}_{1}$ standard deviations above the mean. Condition (ii) requires that either the financial uncertainty shock or the macro uncertainty shock (or both) found in period $\bar{\tau}_{2}$ of September 2008 (the month of the Lehman collapse) be large and exceed $\bar{k}_{2}$ and $\bar{k}_{3}$ standard deviations above the mean, respectively. Condition (iii) requires that the macro uncertainty shock found in period $\bar{\tau}_{3}$ of December 1970 be large and exceed $\bar{k}_{4}$ standard deviations above the mean. Condition (iv) requires that the cumulation of real activity shocks in $\bar{\tau}_{4} \in$ [2007:12,2009:06], corresponding to the Great Recession, be nonpositive, which is to say their sum may not be above average. ${ }^{10}$ Conditions (v) and (vi) are sign restrictions on both types of uncertainty shocks that require they be non-negative during the month $\bar{\tau}_{5}$ of October 1979 (Volcker experiment), and during the months $\bar{\tau}_{6} \in$ [2011:07, 2011:08], corresponding to the 2011 debt-ceiling crisis. This latter episode spills over into two months because the House of Representatives of the U.S. congress did not agree to raise the debt ceiling until July 31 of

\footnotetext{
${ }^{10}$ The dates 2007:12-2009:06 are NBER recession dates, which are taken to be coincident with Great Financial Crisis.
} 
2011, two days prior to when the Treasury estimated the borrowing authority of the United States would be exhausted.

The six event constraints $\bar{g}_{E 1}, \ldots, \bar{g}_{E 6}$ can be represented by a system of inequality constraints on B:

$$
\bar{g}_{E}\left(\mathbf{e}_{t}(\mathbf{B}) ; \overline{\boldsymbol{\tau}}, \overline{\mathbf{k}}\right) \geq 0
$$

where $\overline{\mathbf{k}}=\left(\bar{k}_{1}, \bar{k}_{2}, \bar{k}_{3}, \bar{k}_{4}\right)^{\prime}>0$ are parameters and $\overline{\boldsymbol{\tau}}=\left(\bar{\tau}_{1}, \bar{\tau}_{2}, \bar{\tau}_{3}, \bar{\tau}_{4}, \bar{\tau}_{5}\right)^{\prime}$ are event dates. The choice of the $\overline{\mathbf{k}}$ parameters will be discussed below.

It is important to be clear about what the big shock constraints $\bar{g}_{E 1}, \bar{g}_{E 2}$, and $\bar{g}_{E 23}$ do and do not assume. The restriction $\bar{g}_{E 1}$ stipulates that at least some of the forecast error variance in $\mathbf{X}_{t}$ in 1987:10 must be attributable to a large $e_{F t}$, while $\bar{g}_{E 3}$ stipulates that at least some of the forecast error variance in 1970:12 must be attributable to a large $e_{M t}$. Restriction $\bar{g}_{E 2}$ likewise assumes that at least one of $e_{M t}$ or $e_{F t}$ needs to have been large in the month of the Lehman collapse. What the restrictions do not require is that all or even most of the variation in these episodes be attributable to the specific shocks named in the restriction. In particular, they do not rule out large adverse roles for the other shocks, which are left unrestricted by the constraint. As we shall see below, the Great Financial Crisis/Great Recession was characterized by large adverse shocks in all three variables, even though the second constraint only requires a large role for at least one uncertainty shock in a single month of this episode.

Event constraints put restrictions on the sign and the magnitude of $\mathbf{e}_{t}(\mathbf{B})$ rather than on the signs of impulse responses (IRFs), as is common in some SVAR approaches. Restrictions on the shocks turn out to be valuable for identification because, although two feasible structural models $\mathbf{B}$ and $\tilde{\mathbf{B}}$ will generate shocks $\left\{\mathbf{e}_{t}(\mathbf{B})\right\}_{t=1}^{T}$ and $\left\{\tilde{\mathbf{e}}_{t}(\tilde{\mathbf{B}})\right\}_{t=1}^{T}$ with equivalent first and second moments, $\mathbf{e}_{t}(\mathbf{B})$ and $\tilde{\mathbf{e}}_{t}(\tilde{\mathbf{B}})$ are not necessarily the same at any given $t$. It is not hard to see that if $\mathbf{e}_{t}=\mathbf{Q}^{\prime} \mathbf{P}^{-1} \hat{\boldsymbol{\eta}}_{t}$ and $\tilde{\mathbf{e}}_{t}=\tilde{\mathbf{Q}}^{\prime} \mathbf{P}^{-1} \hat{\boldsymbol{\eta}}_{t}=\tilde{\mathbf{Q}} \mathbf{e}_{t}$, then $\tilde{\mathbf{e}}_{t} \neq \mathbf{e}_{t}$ at any given $t$ when $\tilde{\mathbf{Q}} \neq \mathbf{Q} .{ }^{11}$ Put differently, two series with equivalent properties "on average" can still have distinguishable features in certain subperiods.

B. External Variable Constraints Variables external to the VAR can also facilitate identification when theory or economic reasoning imply they should be informative about the shocks of interest. We use correlations between the external variables and uncertainty shocks to generate additional inequality constraints. ${ }^{12}$ Let $\mathbf{S}_{t}=\left(S_{1 t}, S_{2 t}\right)^{\prime}$ denote a vector of external variables,

${ }^{11}$ Consider the $n=2$ case: $\left(\begin{array}{l}\eta_{1 t} \\ \eta_{2 t}\end{array}\right)=\left(\begin{array}{ll}B_{11} & B_{12} \\ B_{21} & B_{22}\end{array}\right)\left(\begin{array}{l}e_{1 t} \\ e_{2 t}\end{array}\right)$. Solving for $e_{1 t}$ gives $e_{1 t}=|\mathbf{B}|^{-1}\left(B_{22} \eta_{1 t}-B_{12} \eta_{2 t}\right)$, where $|\mathbf{B}|=B_{11} B_{22}-B_{12} B_{21}$ is the determinant of $\mathbf{B}$. The values of $\eta_{1 t}$ and $\eta_{2 t}$ are given by the data. Hence, a restriction on the behavior of $e_{1 t_{1}}$ at specific time $t_{1}$ is a non-linear restriction on $\mathbf{B}$, or equivalently, on $\mathbf{Q}$.

${ }^{12}$ Other researchers have used information in special variables to identify certain effects of uncertainty. Berger, Dew-Becker, and Giglio (2016), while not providing an explicit identification of uncertainty shocks, use options data and find that bad times are associated with higher realized volatility but not higher expected volatility, a result that they interpret as consistent with the hypothesis that higher uncertainty is a consequence rather 
where here $S_{1 t}$ denotes a measure of the aggregate stock market return and $S_{2 t}$ denotes the log difference in the real price of gold. The external variable constraints require that any $\mathbf{e}_{t}(\mathbf{B})$ formed from $\mathbf{B} \in \hat{\mathcal{B}}$ satisfy the following restrictions:

$$
\begin{aligned}
& \text { i } \bar{g}_{C 1}: 0 \geq \operatorname{corr}\left(e_{j t}(\mathbf{B}), S_{1 t}\right), j=M, F \\
& \text { ii } \bar{g}_{C 2}: \operatorname{corr}\left(e_{j t}(\mathbf{B}), S_{2 t}\right) \geq 0, j=M, F
\end{aligned}
$$

The first constraint requires that uncertainty shocks be negatively correlated with stock market returns, and the second requires that they be positively correlated with the log change in the real price of gold. Taken together, the two constraints $\bar{g}_{C 1}$ and $\bar{g}_{C 2}$ can be formulated as a system of inequality constraints:

$$
\bar{g}_{C}(\mathbf{e}(\mathbf{B}) ; \mathbf{S}) \geq 0 .
$$

The external variable constraints provide cross-equation restrictions on the parameters in $\mathbf{B}$. An important aspect of these constraints is that correlations are not invariant to orthonormal rotations. That is to say, correlations generated by $\mathbf{B}$ will in general be different from those generated by $\tilde{\mathbf{B}}=\mathbf{B Q}^{\prime}$.

Two points are worthy of emphasis. First, although the external variables are used to help with identification, they are not required to be valid exogenous instruments, as in the proxyVAR or external IV literature. We discuss this further below. Second, neither gold nor stock market returns are themselves real activity or uncertainty indicators and we are not interested in their behavior per se. This is essential because a central question of our empirical investigation is whether uncertainty shocks are a cause of economic downturns. Identifying restrictions that force this to be true by construction (for example, a requirement that uncertainty shocks be positively related to $S_{3 t}$ where $S_{3 t}$ is the unemployment rate) are not useful because they would require economic downturns characterized by rising unemployment to be at least partly driven by uncertainty shocks.

\subsection{Comparison With Other Methodologies}

The idea of using specific events and/or external variables to identify shocks is not new. Many important studies have used a narrative approach to construct shock series from historical readings of political and economic events to be used as an external IV. The resulting oil price shocks based on timing of wars, tax shocks from fiscal policy announcements, and monetary policy shocks from a reading of FOMC meetings are typically used as though they were exogenous and accurately measured. But as noted in Ramey (2016), both assumptions are questionable. To deal with possible measurement errors, Mertens and Ravn (2014) uses the narrative

than a cause of negative economic shocks. 
tax changes as an external instrument. Similarly, Baker and Bloom (2013) use disaster-like events as instruments for stock market volatility with the aim of isolating exogenous variation in uncertainty. More generally, a prominent proxy-VAR/external IV literature, pioneered by Mertens and Ravn (2013) and Stock and Watson (2008), proposes using variables external to the VAR as instrumental variables to identify SVARs. In all of these papers, point identification is achieved by assuming that the instruments have a zero correlation with some shocks (an exogeneity assumption) and a non-zero correlation with others (a relevance assumption). Like this literature, a maintained assumption of our external variables constraints is that the random processes behind the external variables are determined outside of the VAR system. But unlike this literature, neither our external variables nor our event episodes are presumed to be valid exogenous instruments that have zero correlations with certain shocks. Instead, we only require the weaker assumption that the events and external variables be driven at least in part by one or more of the shocks, thereby allowing us to narrow the set of solutions but not achieve point identification.

Our event constraints differ from the narrative approach in other ways. First, they are data driven rather than being solely based on a narrative reading of history. We use features of the shocks during selected episodes to determine whether a possible solution is admissible. This is tantamount to creating dummy variables from the timing of specific events, and then putting restrictions on their correlation with the identified shocks. Second, the same SVAR is used to identify all shocks simultaneously; it is not a two-step procedure that identifies some shocks ahead of others.

It is worth contrasting the frequentist approach taken here with recent work on signrestricted SVARs in Bayesian contexts. Rubio Ramírez, Waggoner, and Zha (2010) point out that choosing $\mathbf{Q}$ according to the $\mathbf{Q R}$ decomposition amounts to drawing $\mathbf{Q}$ from a uniform distribution over the space of orthogonal matrices. Baumeister and Hamilton (2015) note that an uninformative prior over $\mathbf{Q}$ can be informative for the posterior over the structural impact matrix and impulse responses in sign-restricted SVARs. We differ from these papers in two important ways. First, these papers focus specifically on restrictions placed on the sign of impulse response functions, whereas our restrictions are on timing, magnitude, and correlation, of the shocks. Second, our approach is frequentist in the spirit of the moment inequality framework of Andrews and Soares (2010), with moment conditions given by the inequalities from the event and correlation constraints, and equalities provided by the covariance structure. We use the QR decomposition merely to generate candidate values of $\mathbf{B}$, and check if the resulting $\mathbf{e}_{t}(\mathbf{B})$ satisfies the constraints. Importantly, the shocks that result from our moment inequality restrictions exhibit significant departures from Gaussianity (see discussion below), an outcome that would greatly complicate any Bayesian estimation, but that is straightforward to handle using the frequentist approach of this paper. 
After earlier versions of this paper were circulated, we became aware of work by Antolín-Díaz and Rubio-Ramírez (2018) who, like us, suggest using restrictions on the shocks during certain episodes of history to help identification. They propose several different types of restrictions, including sign restrictions on the shocks at certain dates, as in some of our event constraints above. But there are also several substantive differences. They entertain restrictions that play up the role of some shocks while simultaneously playing down the role of others. (See, for example, their "Type B" restrictions, in which for a particular period or periods the absolute value of one shock's contribution to the unexpected change in a variable is assumed to be larger than the sum of the absolute value of the contributions of all other structural shocks.) This type of restriction differs from the event constraints proposed above because some shocks are presumed to play large roles while others must play smaller or negligible roles, more in the spirit of the traditional narrative-IV approach. By contrast, the event constraints above only require the weaker assumption that the events be driven at least in part by one or more of the shocks; they do not require the remaining shocks to play smaller roles. For example, our restrictions require that there be a large financial uncertainty shock or a large macro uncertainty shock or both in 2008:09, but they do not rule out a large role for the real activity shock during this episode. Other differences are that Antolín-Díaz and Rubio-Ramírez (2018) do not use external variables at all, and their focus is on methodology in a Bayesian context at a general level. While our focus here is to use event and external variable constraints to help understand the role of macro or financial uncertainty in the aggregate economy, the use of shock-based restrictions is not limited to this particular application.

A number of studies have now followed our work to consider macro and financial uncertainty as possibly distinct drivers of fluctuations in real activity. We comment briefly on two here: Carriero, Clark, and Marcellino (2018) and Angelini, Bacchiocchi, Caggiano, and Fanelli (2018). Before doing so, we emphasize that neither of these papers show the shocks implied by their identification schemes. A premise of this paper is that the shocks themselves are important objects of interest, and provide a crucial information for assessing the credibility of any given identification scheme. For example, a scheme that did not imply a fairly large financial uncertainty shock during the 1987 stock market crash is unlikely to be credible, given how isolated this episode was to the stock market.

Carriero, Clark, and Marcellino (2018) specify VARs with uncertainty variables and identify shocks by imposing a parametric specification for their time-varying heteroskedasticity. They include either the JLN macro uncertainty measure $U_{M t}$ or the VIX in a VAR along with several macro variables and conclude that the VIX can arise at least partly as an endogenous response to some macroeconomic events, while macro uncertainty "can be considered exogenous." Numerous differences in modeling assumptions, identification restrictions, and data likely explain our constrasting results. First, our approach doesn't require the imposition of potentially re- 
strictive econometric structure on the conditional moment behavior of the shocks. Instead, all implications flow organically from our constraints, which are straightforward to interpret. For example, our identified shocks are both heteroskedasitic and non-Gaussian, being more volatile in some episodes than others. But this form of heteroskedasticity and non-normality is unlikely to be well captured by any parametric econometric specification of heteroskedasticity. Second, Carriero et. al., place the different uncertainty variables in the VAR one at a time. By contrast, we include them in the VAR together, which forces their effects to be orthogonal. This is important given that the two types of uncertainty are strongly correlated. ${ }^{13}$

Angelini, Bacchiocchi, Caggiano, and Fanelli (2018) use the very same system of variables used here but identify shocks based on zero restrictions in the $\mathbf{B}$ matrix. These restrictions amount to timing restrictions on the dynamics of the variables, which are allowed to vary across subsamples. In particular, they assume that, in subsamples prior to 2008:01 (a break date that is exogenously chosen), $U_{F t}$ shocks could neither contemporaneously affect, nor have been affected by, real activity, i.e., the elements $B_{F Y}$ and $B_{Y F}$ of $\mathbf{B}$ must be jointly zero. They justify these zero restrictions by arguing that financial regulation slowed down the response of financial markets to nonfinancial shocks up to 2008:01. This is an interesting restriction, given that financial markets are typically presumed to respond quickly to news (even in samples prior to 2008), whereas macro variables are comparatively slower moving (e.g., Gertler and Karadi (2015) and Lettau, Ludvigson, and Steindel (2002)). When we apply our procedure on data up to 2008:01, there is no solution in the identified set that satisfies the joint restriction $B_{F Y}=B_{Y F}=0$.

An additional point about the procedure is worth mentioning. The structural shocks we identify do not necessarily correspond to primitive shocks of any particular model, as this is not our goal. Our real activity shocks are 'first moment' shocks that could originate from technology, monetary policy, preferences, or government expenditure innovations. Financial uncertainty, a type of 'second moment' shock, could arise because of expected volatility in financial markets such as fear of a bank run or fear of bankruptcy. Another type of second moment shock, macro uncertainty, could arise because of expected volatility in the macro economy, such as an expectation of greater difficulty in predicting future productivity, future monetary policy or future fiscal policy. An objective of this study is to disentangle whether it is shifts to first or second moments (or both) that drive economic fluctuations. Disentangling the two types of uncertainty is a worthy exercise because the theoretical macro literature on uncertainty has focused on exogenous changes in real activity induced (macro) uncertainty, while the empirical literature has used proxies for macro uncertainty that are highly correlated with volatility in

\footnotetext{
${ }^{13}$ Results (available upon request) show what happens if we apply our procedure to two bivariate VARs where $U_{M t}$ or $U_{F t}$ are included one at a time. Under this estimation, the two measures have very similar dynamic relationships with industrial production, in contrast to our findings when they are simultaneously included in the VAR.
} 
financial markets.

To have confidence in this implementation, we use a simulation to take into account sampling error and study the properties of the estimator. In the Online Appendix, we describe a numerically intensive Monte Carlo simulation that bootstraps from the $\mathbf{e}_{t}(\mathbf{B})$ shocks for the $\mathbf{X}_{t}$ system to create confidence bands for impulse responses. The resulting error bands are reported below for the base case impulse responses.

To summarize, set identification is predicated on three core economic assumptions. First, the identified shocks must be consistent with a priori economic reasoning in a small number of events whose interpretation is relatively incontrovertible. Second, information about uncertainty shocks must be encoded in stock market returns and the real gold price, as specified by the external variable constraints. Third, a maintained assumption of the analysis is that the dynamic responses of interest can be captured without explicitly modeling the random processes behind the external variables.

\section{Data}

We study VAR systems for three systems of data. Our main system is $\mathbf{X}_{t}=\left(U_{M t}, i p_{t}, U_{F t}\right)^{\prime}$, where $U_{M t}$ and $U_{F t}$ are statistical uncertainty indices constructed using the methodology of JLN. Financial uncertainty $U_{F t}$ is new to this paper. In all cases, we use the log of real industrial production, denoted $i p_{t}$, to measure $Y_{t}$. Industrial production is a widely watched economic indicator of business cycles. Subsequent sections consider additional systems that use either policy uncertainty indices in place of $U_{M t}$, or a real activity sub-index of $U_{M t}$ in place of $U_{M t}$. For $S_{1 t}$ we use the Center for Research in Securities Prices (CRSP) value-weighted stock market index return. ${ }^{14}$ For $S_{2 t}$ we use the log difference in the gold price level, deflated using the Consumer Price Index (CPI) with Jan. 2018 as the base month. ${ }^{15}$

Our statistical measures of uncertainty are constructed following the framework of JLN, which aggregates over a large number of estimated uncertainties constructed from a panel of data. Let $y_{j t}^{C} \in Y_{t}^{C}=\left(y_{1 t}^{C}, \ldots, y_{N_{C}}^{C}\right)^{\prime}$ be a variable in category $C$. Its $h$-period ahead uncertainty, denoted by $\mathcal{U}_{j t}^{C}(h)$, is defined to be the volatility of the purely unforecastable component of the future value of the series, conditional on all information available. Specifically,

$$
\mathcal{U}_{j t}^{C}(h) \equiv \sqrt{\mathbb{E}\left[\left(y_{j t+h}^{C}-\mathbb{E}\left[y_{j t+h}^{C} \mid I_{t}\right]\right)^{2} \mid I_{t}\right]}
$$

where $I_{t}$ denotes the information available. Uncertainty in category $C$ is an aggregate of

\footnotetext{
${ }^{14}$ The CRSP index is a value-weighted return of all stocks in NYSE, AMEX, and NASDAQ.

${ }^{15}$ The data source for the CPI-delfated gold price is https://www.macrotrends.net/1333/historical-gold-prices100-year-chart, derived from the London Bullion Market Association (LBMA) measure of daily auction prices of gold, and the Bureau of Labor Statistics.
} 
individual uncertainty series in the category:

$$
U_{C t}(h) \equiv \operatorname{plim}_{N_{C} \rightarrow \infty} \sum_{j=1}^{N_{C}} \frac{1}{N_{C}} \mathcal{U}_{j t}^{C}(h) \equiv \mathbb{E}_{C}\left[\mathcal{U}_{j t}^{C}(h)\right]
$$

If the expectation today of the squared error in forecasting $y_{j t+h}$ rises, uncertainty in the variable increases. As in JLN, the conditional expectation of squared forecast errors in (1) is computed from a stochastic volatility model, while the conditional expectation $\mathbb{E}\left[y_{j t+h}^{C} \mid I_{t}\right]$ is replaced by a diffusion index forecast, augmented to allow for nonlinearities. These are predictions of an autoregression augmented with a small number of common factors estimated from a large number of economic time series $x_{i t}$ assumed to have factor structure. Nonlinearities are accommodated by including polynomial terms in the factors, and factors estimated from squares of the raw data. The use of large datasets reduces the possibility of biases that arise when relevant predictive information is ignored.

In this paper, we consider two categories of uncertainty, macro $M$ and financial $F$. Hence there are two datasets, both covering the sample 1960:07-2015:04. For macro uncertainty $U_{M t}$, we use a monthly macro dataset, $\mathcal{X}_{t}^{M}$, consisting of 134 mostly macroeconomic time series taken from McCracken and $\mathrm{Ng}$ (2016). For financial uncertainty $U_{F t}$, we use a financial dataset $\mathcal{X}_{t}^{F}$ consisting of 148 measures of monthly financial indicators. ${ }^{16}$ We also use two measures of policy uncertainty taken from Baker, Bloom, and Davis (2016) in lieu of the statistical measure of macro uncertainty $U_{M t}$.

The 134 macro series in $\mathcal{X}^{m}$ are selected to represent broad categories of macroeconomic time series. The majority of these are real activity measures: real output and income, employment and hours, real retail, manufacturing and trade sales, consumer spending, housing starts, inventories and inventory sales ratios, orders and unfilled orders, compensation and labor costs, and capacity utilization measures. The dataset also includes commodity and price indexes and a handful of bond and stock market indexes, and foreign exchange measures. The financial dataset $\mathcal{X}^{f}$ is an updated monthly version of the of 148 variables comprised solely of financial market time series used in Ludvigson and Ng (2007). These data include valuation ratios such as the dividend-price ratio and earnings-price ratio, growth rates of aggregate dividends and prices, default and term spreads, yields on corporate bonds of different ratings grades, yields on Treasuries and yield spreads, and a broad cross-section of industry, size, book-market, and momentum portfolio equity returns. ${ }^{17}$ The indexes $U_{M t}$ and $U_{F t}$ lend themselves to different interpretations because they are constructed from different variables. In a subsequent section, we investigate the sub-index of $U_{M t}$ that is constructed only from the real activity variables.

\footnotetext{
${ }^{16}$ Both datasets were previously used in Ludvigson and $\mathrm{Ng}$ (2007) and JLN, but they are updated to the longer sample.

${ }^{17}$ A detailed description of the series is given in the Data Appendix of the online supplementary file at www.sydneyludvigson.com/s/ucc_data_appendix.pdf
} 
The top panel of Figure 1 plots the estimated $h=1$ month-ahead macro uncertainty, denoted simply $U_{M t}$, in standardized units along with the NBER recession dates. The horizontal bar corresponds to 1.65 standard deviation above unconditional mean of each series (which is standardized to zero). As is known from JLN, the macro uncertainty index is strongly countercyclical, and exhibits large spikes in the deepest recessions. The updated data $U_{M t}$ series shows much the same. Though $U_{M t}$ exceeds 1.65 standard deviations 48 times, they are clustered around the 1973-75 and 1981-82 recessions, as well as the Great Recession of 2007-09. Macroeconomic uncertainty has a correlation of -0.65 with the 12 -month moving-average of the growth in industrial production.

The middle panel of Figure 1 plots the estimated $h=1$ month-ahead financial uncertainty series, denoted $U_{F t}$, over time, which is new to this paper. $U_{F t}$ is a broad-based measure of time varying financial uncertainty using data from the bond market, stock market portfolio returns, and commodity markets. As seen from Figure 1, $U_{F t}$ is also countercyclical, though less so than $U_{M t}$. Its correlation with industrial production is -0.39 . The series often exhibits spikes around the times when $U_{M t}$ is high. However, $U_{F t}$ is more volatile and spikes more frequently outside of recessions, the most notable being the 1987 stock market crash. Though observations on $U_{F t}$ exceed the 1.65 standard deviation line 33 times, they are spread out in seven episodes, with the 2008 and 1987 episodes being the most pronounced.

As is clear from Figure 1, both indicators of macro and financial uncertainty are serially correlated and hence predictable. They have comovements but also have independent variations as the correlation between them is only 0.58. However, this unconditional correlation cannot be given a structural interpretation. To the extent that our uncertainty variables measure expectations about future volatility, the heightened uncertainty measures can respond endogenously to events that are expected to happen, but they can also be exogenous changes to expected volatility. We use a VAR to capture the predictable variations, and then identify uncertainty shocks from the VAR residuals using the restrictions described above. We now turn to the implementation issues.

\section{Implementation}

Estimates of $\mathbf{B}$ that satisfy the reduced form covariance restrictions, the event constraints, and the external variable constraints together give the identified solution set denoted

$$
\begin{aligned}
\overline{\mathcal{B}}(\mathbf{B} ; \overline{\mathbf{k}}, \overline{\boldsymbol{\tau}}, \mathbf{S})= & \left\{\mathbf{B}=\hat{\mathbf{P}} \mathbf{Q}: \mathbf{Q} \in \mathbb{O}_{n}, \quad \operatorname{diag}(\mathbf{B})>0 ;\right. \\
& \left.\bar{g}_{Z}(\mathbf{B})=0, \bar{g}_{E}(\mathbf{B} ; \bar{\tau}, \overline{\mathbf{k}}) \geq 0, \bar{g}_{C}(\mathbf{B} ; \mathbf{S}) \geq 0\right\} .
\end{aligned}
$$

To simplify notation, we simply write $\overline{\mathcal{B}}(\mathbf{B} ; \overline{\mathbf{k}}, \overline{\boldsymbol{\tau}}, \mathbf{S})$ as $\overline{\mathcal{B}}$. The unconstrained set $\hat{\mathcal{B}}$ is constructed using random rotations, as discussed above. A particular solution can be in both $\hat{\mathcal{B}}$ and $\overline{\mathcal{B}}$ only 
if all the event and external variable constraints are satisfied.

Though no one solution in $\overline{\mathcal{B}}$ is any more likely than another, we sometimes use what will be referred to as the 'maxG' solution as reference point:

$$
\mathbf{B}^{\max G} \equiv \underset{\mathbf{B} \in \overline{\mathcal{B}}}{\arg \max } \bar{g}_{C}(\mathbf{B})^{\prime} \bar{g}_{C}(\mathbf{B}) .
$$

This is the single solution in the identified set for which the inequalities pertaining to the external variable constraints are collectively maximized, as measured by an equally-weighted quadratic norm. One may consider this the solution for which the external variables are maximally relevant/informative for the shocks of interest.

Construction of the identified solution necessitates choice of the big shock constraint parameters $\mathbf{k}=\left(\bar{k}_{1}, \bar{k}_{2}, \bar{k}_{3}, \bar{k}_{4}\right)^{\prime}$. It should be clear that if the values for these parameters are overly restrictive, the identified solution set will be empty. If they are too unrestrictive, the constraints will have no identifying power. Shock-based restrictions are also not invariant to the system being analyzed because the data may have different variability, as well as different skewness, kurtosis, and cross-moments. Thus the parameters for one system of data could be too restrictive for another. We therefore set baseline values for $\overline{\mathbf{k}}$ using a data dependent procedure for the system $\mathbf{X}_{t}=\left(U_{M t}, i p_{t}, U_{F t}\right)^{\prime}$ so they are consistent with the economic reasoning behind the constraints, and then investigate the sensitivity of results to changes in this parameterization. These parameters can then be adapted to other systems. We now discuss the parameterization for the system $\mathbf{X}_{t}=\left(U_{M t}, i p_{t}, U_{F t}\right)^{\prime}$.

The event constraints $\bar{g}_{E 1}, \bar{g}_{E 2}$, and $\bar{g}_{E 3}$ require financial and/or macro uncertainty shocks to be large in the month October of the 1987 crash, the month September of the 2008 Lehman collapse, and in the month December of high real uncertainty shocks in 1970. But what constitutes a large shock in this system? To address this question, we return to the unconstrained set $\hat{\mathcal{B}}$ for the system $\mathbf{X}_{t}=\left(U_{M t}, i p_{t}, U_{F t}\right)^{\prime}$. In our sample, the largest shocks to $U_{F t}$ in $\hat{\mathcal{B}}$ using $h=1$ month uncertainty are typically above four standard deviations, and similarly for $U_{M t}$. The 75th-percentile value of $e_{F t}$ in 1987:10 is 4.16, and is 4.57 in 2008:09. The 75th-percentile value of $e_{M t}$ in 1970:12 is 4.05 and is 4.73 in 2008:09. If shocks were Gaussian, the probability of a shock of these magnitudes would be less than 1.3e-4. But as we show below, the identified shocks are non-Gaussian and exhibit substantial excess skewness and leptokurtosis. As a baseline, we set $\overline{\mathbf{k}}=\left(\bar{k}_{1}, \bar{k}_{2}, \bar{k}_{3}, \bar{k}_{4}\right)^{\prime}$ equal to their respective 75 th percentile values, thereby requiring a "big" shock to be in the top $25 \%$ of all shocks in $\hat{\mathcal{B}}$ during those episodes. Estimates for the baseline system $\mathbf{X}_{t}=\left(U_{M t}, i p_{t}, U_{F t}\right)^{\prime}$ using these values will be referred to as our base case. Below we assess the sensitivity of the results to this parameterization by requiring big shocks for these episodes to merely exceed their respective median values in the unconstrained set.

It is worth noting that both 1970 and 1987 event constraints alone eliminate $75 \%$ of the 
solutions, while the Lehman event constraint alone eliminates 90\%. These event constraints together with the constraint that the cumulative sum of real activity shocks in the Great Recession should not be above average eliminate $99 \%$ of the solutions in $\hat{\mathcal{B}}$. Of course one percent of 1.5 million draws is still a non-trivial number. But when all event constraints are combined with the correlation constraints, we are left with 169 accepted draws, which is $25 \%$ of the sample size.

\section{Results}

This section presents our main results. The first subsection considers the base case empirical model, which uses the macro uncertainty index $U_{M t}$; the next subsection considers a VAR in which policy uncertainty is used in place of the macro uncertainty index $U_{M t}$. The subsequent section discusses additional analyses and sensitivity checks pertaining to the parameterization of the base case. We focus in this section on studying one-month-ahead uncertainty $h=1$, the horizon over which uncertainty displays the most variability (see LMN). Longer horizon uncertainty will be considered in a subsequent section. We use $p=6$ lags in the VARs, noting that using 12 lags makes no difference to the results.

Before getting into the main results, its worth commenting on the second event constraint, which requires either a large financial uncertainty shock or a large macro uncertainty shock (or both) in $\bar{\tau}_{2}=2008: 09$, the month of the Lehman collapse. An examination of the unconstrained solution set shows that no solutions have large values for both $e_{F \bar{\tau}_{2}}$ and $e_{M \bar{\tau}_{2}}$ in 2008:09, where large is defined as being in the top $25 \%$ of their respective distributions in that period. Thus any identified subset of $\hat{\mathcal{B}}$ can only contain solutions in which one or the other is large in that month. The estimation procedure must therefore choose which uncertainty shock was large. Among all solutions in the base case identified set, $100 \%$ of them place the big uncertainty shock in $U_{F t}$, rather than $U_{M t}$ in 2008:09. That is, the estimation always choose $e_{F \bar{\tau}_{2}}$ to be big (in the top $25 \%$ of all $e_{F \bar{\tau}_{2}}$ in the unconstrained set, or at least 4.6 standard deviations), while the values for $e_{M \bar{\tau}_{2}}$ are estimated to range from 0.43 to 2.1 standard deviations. As we shall see below, however, other months of the GFC had values for $e_{M t}$ that were large, many greater than 4 standard deviations, so the episode as a whole is characterized by large uncertainty shocks of both types.

\subsection{Systems with Macro Uncertainty}

This subsection considers the baseline system $\mathbf{X}_{t}=\left(U_{M t}, i p_{t}, U_{F t}\right)^{\prime}$ under with the base case parameter values.

To get a sense of the behavior of the shocks in this system, the left panel of Figure 2 presents the time series of the standardized shocks $\left(e_{M}, e_{i p}, e_{F}\right)$ for the maxG solution. All 
three types of shocks display strong departures from normality with excess skewness and/or excess kurtosis. The largest of the positive $e_{i p}$ shocks is recorded in 1971:01 followed by 2005:10, while the largest of the negative $e_{i p}$ shocks is recorded in 2008:09, followed by 1980:04. There also appears to be a moderation in the volatility of the ip shocks in the post-1983 period. For this particular solution, the largest positive $e_{M}$ shock is in 1970:12 (run-up to Bretton Woods collapse), followed by the shock in 2008:10 (the month after the Lehman collapse). The largest positive $e_{F}$ shock is recorded in 2008:09 (month of the Lehman collapse) followed by 1987:10 (Black Monday). For $e_{F}$, the extreme but transitory nature of the 1987 stock market crash leads to a very large spike upward in $e_{F}$ in the month of the crash, followed by a very large spike downward in the month following the crash as the market recovered strongly and quickly. While this episode magnifies the spike in $e_{F}$ in 1987, it is largely orthogonal to real activity and macro uncertainty. Observe that the large $i p$ shock in 2005:10 is not associated with a contemporaneous spike in uncertainty, while there are several spikes in both types of uncertainty that do not coincide with spikes in $e_{i p}$.

We now focus on large "adverse" shocks, namely large positive uncertainty shocks and large negative real activity shocks recovered by the econometric methodology. The right panel of Figure 2 displays the date and size of all $e_{M t}$ and $e_{F t}$ shocks in the identified set that are at least two standard deviations above the mean and all negative $e_{i p t}$ shocks exceeding two standard deviations. In view of the non-normality of the shocks, the figure also plots horizontal lines corresponding to three standard deviation of the unit shocks, which is used as the reference point for "large."

The right panel of Figure 2 shows that the solutions identify big financial uncertainty shocks in October 1987 and in one or more months of 2008. Such solutions are selected as part of the identification scheme. The middle panel shows that large negative real activity shocks are in alignment with all post-war recessions with one exception: the negative real activity shock in 2005 is not immediately associated with a recession, but it could be the seed of the Great Recession that followed. It's clear that parts of the real economy were showing signs of deterioration prior to the onset of the recession as dated by the NBER. For example, it is known that the housing market led the 2007-2009 recession (e.g., see Favilukis, Ludvigson, and Van Nieuwerburgh (2017) for a discussion). Indeed, all 10 housing series in $\mathcal{X}^{M}$ (most pertaining to housing starts and permits series) exhibit sharp declines starting in September 2005 and continuing through 2006, thereby leading the Great Recession.

The right panel of Figure 2 shows that the dates of large increases in $e_{M}$ are less clustered. They generally coincide with, or occur shortly after, the big real activity shocks and the financial uncertainty shocks. Large macro uncertainty shocks occurred more frequently in the pre-1983 than the post 1983 sample, consistent with a Great Moderation occurring over the period ending in the Great Recession. 
Although our event restrictions require that large financial uncertainty shocks play an important role in the 1987 crash and in 2008 in the month of the Lehman collapse, they by no means rule out large adverse roles for the other shocks in the GR/GFC. In particular, our restrictions do not require that all or even most of the variation in these episodes be attributable to shocks that originated in financial markets. The right panel of Figure 2 shows many large adverse values of $e_{M t}$ and $e_{i p t}$ in the Great Financial Crisis/Great Recession episode. Indeed, all of the solutions in the identified set under the baseline parameters have an $e_{M t}$ greater than three standard deviations above the mean and an an $e_{i p t}$ less than -3 standard deviations below the mean in at least one month of the 2007-09 Great Recession/financial crisis. It is a result of the analysis that there were big shocks everywhere in this episode. These big shock episodes are partly responsible for the non-Gaussian aspects of the shocks. It would be desirable for dynamic equilibrium models that wish to study the effects of uncertainty to incorporate shocks with such non-Gaussian features.

\subsubsection{Impulse Response Functions}

We now use impulse response functions to better understand the dynamic causal effects and propagating mechanisms of the shocks. Figure 3 shows, for our base case system, the identified set of dynamic responses, or impulse response functions (IRFs), of each variable in the SVAR to a one standard deviation increase in each of structural shocks. The solid lines report the impulse responses for all solutions in the identified set $\overline{\mathcal{B}}$. The dotted line show $95 \%$ confidence sets for the identified set of responses. Several results stand out.

First, positive shocks to financial uncertainty $e_{F}$ (center plot, bottom row) lead to a sharp decline in production that persists for many months. All solutions that satisfy the identification restrictions have this pattern and the identified set of responses is bounded well away from zero as the horizon increases. Positive perturbations to $e_{F t}$ also cause $U_{M t}$ to increase sharply (third row). These results lend support to the hypothesis that heightened financial uncertainty is an exogenous impulse that causes declines in real activity. However, there is little evidence that heightened financial uncertainty is a result of lower economic activity. Instead, positive shocks to production increase financial uncertainty.

The IRFs show responses to one standard deviation shocks in financial uncertainty. But the identification strategy uses big shock events in financial uncertainty to identify the shocks. It is worth emphasizing that, for the many $e_{F t}$ in these episodes that are identified to be four or more standard deviations above zero, the effects of the shocks shown in Figure 3 would be many times larger than that shown. A one-standard deviation increase in $e_{F t}$ reduces $i p$ by $1 \%$ after 60 months, according to the median response at that horizon. This decline represents about a 1.3 standard deviation change in monthly ip growth. So a four standard deviation shock would reduce $i p$ by $4 \%$, which represents a 5.2 standard deviation change in monthly $i p$ 
growth. Infrequent big shocks, when they hit, can have large effects.

Second, while we find no evidence that high financial uncertainty is a consequence of lower economic activity, the results for macro uncertainty are quite different. Macro uncertainty falls sharply in response to positive $i p$ shocks. Alternatively stated, negative $i p$ shocks cause macro uncertainty to increase sharply. These endogenous movements in macro uncertainty persist for about five years after the real activity shock, a result that is strongly apparent in all the solutions of the identified set.

Third, there is little evidence that the observed negative correlation between macro uncertainty and real activity is the result of positive macro uncertainty shocks that drive down production. The top middle panel shows that all solutions in the identified set imply that positive macro uncertainty shocks increase real activity in the short run, consistent with growth options theories discussed above. All solutions in the identified set imply that production is reduced in the long-run, though the confidence set widens as the horizon extends. These findings suggest that higher macro uncertainty in recessions is a response to lower economic activity rather than a causal factor in recessions.

The finding that $U_{M}$ responds endogenously to other shocks implies that macro uncertainty could be an important amplification factor in recessions even if it doesn't cause them. Figure 3 shows that $U_{M}$ rises sharply in response to either a negative $i p$ shock or a positive $U_{F}$ shock. The impact of these adverse shocks on the economy might be far less if $U_{M}$ didn't respond to them in the first place.

To investigate this possibility, we consider a set of IRFs where $B_{M Y}=B_{M F}$ are restricted to 0 (in contrast to our estimates) while all other elements of $\mathbf{B}$ are maintained at the base case estimated values. This experiment shuts off the impact effect on $U_{M}$ of the other shocks in the system, implying that recessions caused by a negative first moment shock or a positive second moment shock to financial uncertainty are presumed, hypothetically, to have no effect initially on macro uncertainty. Figure 4 shows the set of hypothetical IRFs, juxtaposing them against the set of base case responses. Comparing just the median values of the set of hypothetical IRFs with those for the base case, we find that the effect of adverse shocks on industrial production is much smaller under the hypothetical than under the base case estimates. Specifically, the effect of an adverse ip shock on ip five years out is dampened by $68 \%$ under the hypothetical compared to the base case, while the effect of an adverse $U_{F}$ shock on $i p$ five years out is dampened by $28 \%$. These findings suggest that higher macro uncertainty in recessions plays an important role in amplifying adverse first-moment shocks as well as second-moment shocks with origins in financial markets. 


\subsubsection{Decomposition of Variance}

To give a sense of the historical importance of these shocks, we perform a decomposition of variance for each solution in the identified set. We report the fraction of $s$-step-ahead forecast error variance attributable to each structural shock $e_{M t}, e_{i p t}$, and $e_{F t}$ for $s=1, s=12, s=\infty$, and $s_{\max }$, where $s_{\max }$ is the horizon at which the fraction of forecast error variance is maximized. Because we have a set of solutions, we have a range of forecast error variances for each $s$. The left panel of Table 1 reports the range of values for the $\mathbf{X}_{t}$ system. The right panel of Table 1 are results for an alternative measure of uncertainty and will be discussed below.

According to the top row, real activity shocks $e_{i p t}$ have sizable effects on macroeconomic uncertainty $U_{M}$, with the fraction of forecast error variance ranging from 0.52 to 0.72 at the $s_{\max }$ horizon. But according to the bottom row, these same shocks have small effects on financial uncertainty $U_{F t}$, with a range of forecast error variance from 0.02 to 0.10 at horizon $s_{\max }$. The middle row shows that positive macro uncertainty shocks $e_{M}$, which increase rather than decrease real activity, explain a surprisingly large fraction of production, with effects at $s_{\max }$ horizon ranging from 0.37 to 0.62 .

Though financial uncertainty shocks $e_{F t}$ have a small contribution to the one-step-ahead forecast error variance of $i p_{t}$, their relative importance increases over time so that they account for 0.38 to 0.54 of the forecast error variance in $i p$ at the $s_{\max }$ horizon. Financial uncertainty is unlike macro uncertainty or real activity in that its variation is far more dominated by its own shocks. As seen from Table 1, $e_{F t}$ shocks explain between 0.84 and 0.94 of the $s=1$ step-ahead forecast error variance in $U_{F t}$, and between 0.70 and 0.86 at the $s=\infty$ horizon. At the $s_{\max }$ horizon, the range of forecast error variance is 0.84 to 0.94 .

To summarize, positive real activity shocks $e_{i p t}$ have quantitatively large persistent and negative effects on macro uncertainty $U_{M t}$. In turn, positive macro uncertainty shocks $e_{M t}$ have positive effects on production, especially in the short-run. By contrast, positive financial uncertainty shocks $e_{F t}$ have large negative effects on production, especially in the long run. Across all VAR forecast horizons, the forecast error variance of financial uncertainty is the least affected by shocks other than its own, implying that $U_{F t}$ is quantitatively the most important exogenous impulse in the system.

\subsection{Systems with Policy Uncertainty}

The results above suggest that the dynamic relationship between macro uncertainty and real activity may be quite different from the relation between financial uncertainty and real activity. However, given the composition of our macro data, macroeconomic uncertainty itself can be due to uncertainty in real activity variables such as output and unemployment, to price variables, and to financial market variables. The theoretical uncertainty literature has focused 
on modeling exogenous uncertainty shocks that arise specifically in measures of real economic fundamentals, rather than in prices or financial markets. To better evaluate the implications of these theoretical models and to examine robustness to alternative measures of macro or real economic uncertainty, we repeat our analysis using the economic policy uncertainty (EPU) indices of Baker, Bloom, and Davis (2016) (BBD), in place of the macro uncertainty index $U_{M t}$. $\mathrm{BBD}$ find that firms with greater exposure to government expenditures reduce investment and employment growth when policy uncertainty rises, suggesting that the EPU indices are well characterized as measures of real economic uncertainty.

BBD compute two EPU indices, a "baseline" EPU index that has three components, and a news-only index that is a subindex and one component of the baseline EPU index. We denote these the EPU and EPN index, respectively. These indices are available from 1987:01 to 2017:06. We then repeat the analysis for two systems: $\mathbf{X}_{t}^{E P U}=\left(E P U_{t}, i p_{t}, U_{F t}\right)^{\prime}$, and $\mathbf{X}_{t}^{E P N}\left(E P N_{t}, i p_{t}, U_{F t}\right)^{\prime}$.

The left panel of Figure 5 plots the EPU and EPN indices over time. We observe that the two largest spikes upward in the baseline index are in and just after the debt ceiling crisis resolution, which correspond to the dates 2011:07 and 2011:08. For news index, there is an additional spike upward that rivals these in size: that for September 11, 2001. An inspection of the unconstrained set for these systems reveals that both episodes, but especially the debt ceiling crisis of 2011, were far bigger uncertainty events for policy uncertainty shocks than for either macro or financial uncertainty shocks. In other words, the debt ceiling crisis is the big shock event for policy uncertainty, akin to the 1987 crash and the Lehman collapse for financial uncertainty. We use these events to help with identification in the policy uncertainty systems.

To facilitate comparability with the baseline system, we normalize $E P U_{t}$ and $E P N_{t}$ to have the same mean and standard deviation as $U_{M t}$. The constraints $\bar{g}_{E 1}, \bar{g}_{E 2}, \bar{g}_{E 3}$, used above on $e_{F t}(\mathbf{B})$ and $e_{i p t}(\mathbf{B})$ are maintained in these systems, while the external variable constraints $\bar{g}_{C 1}$ and $\bar{g}_{C 2}$ are now maintained for $e_{F t}(\mathbf{B})$ as well as for the policy shocks $e_{E P U t}(\mathbf{B})$ and $e_{E P N t}(\mathbf{B})$. The parameters for these constraints are set as described above for the base case. The previous event constraints $\bar{g}_{E 4}$ (Volcker policy announcement) is not contained in the sample for this system, so it is dropped and replaced by an inequality constraint for September 11th, 2001. The new event constraints for this system are parameterized as follows:

\begin{tabular}{l|cl|l} 
Constraint & \multicolumn{1}{|c|}{$\mathbf{X}_{t}^{E P U}$} & \multicolumn{1}{c}{$\mathbf{X}_{t}^{E P N}$} & \multicolumn{1}{c}{$\tau$} \\
\hline $\bar{g}_{E 4}:$ & - & $e_{E P N \bar{\tau}_{4}}(\mathbf{B}) \geq 0$ & for $\bar{\tau}_{4}=2001: 09$ \\
$\bar{g}_{E 5}:$ & $e_{E P U \bar{\tau}_{5}}(\mathbf{B})-2 \geq 0$ & $e_{E P N \bar{\tau}_{5}}(\mathbf{B})-2 \geq 0$ & for $\bar{\tau}_{5}=[2011: 08,2011: 09]$ \\
\hline
\end{tabular}

Constraint $\bar{g}_{E 4}$ requires the news uncertainty shock to merely be above average in the month of 2011 terrorist attacks. Constraint $\bar{g}_{E 5}$ requires both types of policy shocks to be large, i.e., bounded away from zero, in the months of the debt ceiling crisis. An inspection of the unconstrained set for these systems shows that a two standard deviation policy uncertainty shock in 
the $\mathbf{X}_{t}^{E P U}$ and $\mathbf{X}_{t}^{E P N}$ systems is roughly comparable to a non-negative macro uncertainty shock in the base case system. That is, two standard deviations corresponds to the 65th percentile value in the unconstrained set of the minimum $e_{E P N \bar{\tau}_{4}}(\mathbf{B})$ over the two months in the debt ceiling crisis, while zero standard deviations corresponds to same percentile value for $e_{M t}(\mathbf{B})$ in the debt ceiling crisis in the base case system.

The right panel of Figure 5 shows the dynamic responses for the $\mathbf{X}_{t}^{E P U}$ and $\mathbf{X}_{t}^{E P N}$ systems. The character of the responses is similar to those for the systems based on the JLN uncertainty measures. Policy uncertainty falls sharply in response to positive production shock. Alternatively stated, negative shocks to production increase policy uncertainty sharply. These endogenous movements in policy uncertainty are more transient than those to macro uncertainty, however, and are eliminated in about two years. Financial uncertainty shocks in this system continue to be a driving force for real activity, with positive shocks driving down $i p_{t}$ sharply and persistently. But there is no evidence that positive shocks to $i p_{t}$ drive down financial uncertainty; in fact such shocks drive financial uncertainty persistently upward. There is no evidence based on the either system that positive policy uncertainty shocks drive down real activity; the opposite is found, with positive shocks to policy uncertainty driving up production even more persistently than in the $\mathbf{X}_{t}$ system. These findings reinforce the previous results that countercyclical increases in real economic uncertainty are often well characterized as endogenous responses to declines in real activity, rather than exogenous impulses driving real activity downward, while the opposite is true for financial uncertainty. Interestingly, positive shocks to policy uncertainty drive financial uncertainty down, suggesting that markets may view times of high policy uncertainty as upside rather than downside risk.

To complete the analysis, we present variance decompositions for the $\mathbf{X}_{t}^{E P U}$ system (the results for the system $\mathbf{X}_{t}^{E P N}$ are similar). These results, presented in the right panel of Table 1 , share some similarities with the $\mathbf{X}_{t}$ system shown in the left panel, but there are at least two distinctions. First, financial uncertainty shocks that decrease real activity in both systems explain a smaller fraction of the forecast error variance in production in the $\mathbf{X}_{t}^{E P U}$ system at all but the $s=1$ forecast horizon. The ranges for these numbers at the $s=s_{\max }$ horizon across all solutions in the identified set are $[0.27,0.39]$ in the $\mathbf{X}_{t}^{E P U}$ system compared to $[0.38,0.54]$ in the $\mathbf{X}_{t}$ system. Second, compared to the $\mathbf{X}_{t}$ system, greater fractions of the forecast error variance in $U_{F t}$ are explained by $i p$ shocks. That is likely because positive shocks to production have more persistent effects on financial uncertainty in the $\mathbf{X}_{t}^{E P U}$ system. $^{18}$

\footnotetext{
${ }^{18}$ It is worth noting that the results for the EPU systems are very similar even if no correlation constraints with $S_{t}$ are imposed. For these systems, the event constraints alone appear to be sufficient for identifying the dynamic relationships in the system.
} 


\section{$7 \quad$ Alternative Specifications}

This section considers several alternative specifications, including alternative parameterizations of the base case big shock constraints, alternative identifying restrictions, a system that uses uncertainty in real activity variables rather than that for all macroeconomic series, an evaluation of the validity of recursive identification restrictions, and longer horizon uncertainty.

\subsection{Alternative Parameterization of Big Shock Events}

In the base case specification, we set $\overline{\mathbf{k}}=\left(\bar{k}_{1}, \bar{k}_{2}, \bar{k}_{3}, \bar{k}_{4}\right)^{\prime}$ equal to the 75 th-percentile values of $e_{F t}$ and/or $e_{M t}$ implied by the 1.5 million values of $\mathbf{B}$ in the unconstrained set $\hat{\mathcal{B}}$ in dates $\bar{\tau}_{1}, \bar{\tau}_{2}, \bar{\tau}_{2}$, and $\bar{\tau}_{3}$, respectively, thereby requiring a "big" shock to $e_{j t}, j=M, F$ to be in the top $25 \%$ of all observed $e_{j t}$ in $\hat{\mathcal{B}}$ for that date. We now slacken the these constraints by setting $\overline{\mathbf{k}}$ so that a large shock in these episodes needs to merely exceed their respective median values, while keeping all other parameters at their base case values. Under this parameterization a "large" financial uncertainty shock need be only be 2.8 rather than 4.2 standard deviations above the mean in 1987:10, and need be only 1.9 standard deviations above the mean rather than 4.6 in 2008:09. Likewise a "large" macro uncertainty shock need be only be 2.3 rather than 4 standard deviations above the mean in 1970:12, and need be only 1.9 standard deviations above the mean rather than 4.7 in 2008:09.

The left panel of Figure 6 shows the IRFs when $\bar{k}_{1}$ and $\bar{k}_{4}$, the parameters governing the size of big shock events in 1987:10 (stock market crash) and 1970:12 (run-up to Bretton Woods collapse), are simultaneously slackened to their respective median values of $\hat{\mathcal{B}}$, while all other parameters are held fixed at their baseline values. The right panel of Figure 6 shows the IRF when $\bar{k}_{2}$ and $\bar{k}_{3}$, the parameters governing the size of the big shock event for $U_{F t}$ and/or $U_{M t}$ in 2008:09 (Lehman collapse) are simultaneously slackened to their respective median values of $\hat{\mathcal{B}}$, while all other parameters are held fixed at their baseline values. In both panels, the identified set under the baseline parameterization is superimposed on the figure for comparison.

Figure 6 shows that, while the bounds of the identified set are inevitably wider given the weaker constraints, they nevertheless remain informative, with the qualitative results effectively the same as for the base case. Thus, the findings reported above for the base case are not highly sensitive to the definition of what constitutes "big" in the big shock constraints. In fact, fixing other parameters, the bounds remain informative if the "big" shock in the Lehman episode is required to be just 1.7 standard deviations, while those in 1987:10 and 1970:12 are required to be merely non-negative.

It is worth noting that we do not find any alternative parameterizations for these parameters in which clear conclusions emerge that are of an entirely different nature from those of the base case. Either the constraints are slackened to a point where they have little identifying power, 
leaving the bounds uninformative, or the constraints are tightened to a point where they are incompatible with the data, leaving the identified set empty, or the qualitative results are the same as reported for the base case. No matter what the parameters, there is little basis under these identifying restrictions for concluding that positive macro uncertainty shocks cause declines in production, at least initially. By contrast, the evidence that macro uncertainty amplifies the economic consequences of adverse shocks is robust to a range of parameterizations of the base case constraints.

\subsection{Alternative Restrictions}

A key finding from the base case system is that macro and financial uncertainty have very different dynamic causal relationships with production, a result that is especially evident from the opposite-signed impact responses of production to the two types of uncertainty shocks. A positive financial uncertainty shock drives down production, while a positive macro uncertainty shock drives it up. These impact responses may be read off from the off-diagonal elements of our estimated $\mathbf{B}$ matrices, where $B_{Y M}$ denotes the impact response or $Y$ to an $e_{M}$ shock, and $B_{Y F}$ denotes the impact response of $Y$ to an $e_{F}$ shock. We now investigate which of our identifying restrictions are responsible for these results.

The left panel of Figure 7 displays the distribution of values for $B_{Y M}$ and $B_{Y F}$ under three different sets of identifying restrictions: (i) for all values in the unconstrained set, which imposes only the reduced form covariance restrictions (blue histogram), (ii) for all values in an identified set that only imposes the covariance restrictions and event constraints but not the external variable constraints (red histogram), and (iii) for all values in the base case identified set, which imposes all constraints, including covariance, event and external variable constraints (black histogram). Notably, the covariance/event-only constrained solutions for $B_{Y F}$ are all negative while those for $B_{Y M}$ are all positive, as they are for the base case. By contrast, in the unconstrained set the distributions for both parameters contain both positive and negative values. Thus, the event constraints alone are enough to pin down the differing signs of these parameters. Once we add the external variable constraints to the event and covariance restrictions, the distribution of $B_{Y M}$, with all positive values, is shifted more toward zero, while the distribution of $B_{Y F}$, with all negative values, is shifted more away from zero. Thus, the external variables constraints prevent the response of production to a positive macro uncertainty shock from being too positive, and prevent the response of production to a positive financial uncertainty shock from being too small in absolute value. In short, the event constraints restrict the signs of $B_{Y F}$ and $B_{Y M}$, while the external variable constraints restrict their magnitudes.

The right panel of Figure 7 shows analogous histograms under another alternative set of restrictions: (i) that for the unconstrained set (blue histogram), (ii) that for an identified set 
if we impose all base case constraints except the Lehman event constraint (red histogram), and (iii) that for the identified set under all base case constraints (black histogram). What is clear from this plot is that the Lehman event is crucial for obtaining informative bounds for the impact response of production to a financial uncertainty shock. Without the Lehman event constraint, the distribution of values for $B_{Y F}$ contains both positive and negative values. The distribution of values for $B_{Y M}$, however, continues to contain only positive values even without the Lehman event constraint, though the range of positive values is much wider than for the base case. A premise of this paper is that the 2007-09 financial crisis and Lehman collapse was an important rare event that can help distinguish the transmission of financial versus real uncertainty shocks. This maintained assumption is supported by these results and those for the alternative parameterizations of $\overline{\mathbf{k}}$ displayed in the previous subsection. Taken together, they show that, if the identified set includes solutions for which the Lehman collapse is not accompanied by at least a modestly sized uncertainty shock of one type or another, the estimation cannot distinguish their separate roles. Such solutions are ruled out by our identifying assumptions.

\subsection{Systems with Real Activity Uncertainty}

As noted, $U_{M t}$ can fluctuate due to uncertainty in real activity variables such as output and unemployment, or due to price variables or financial market variables. In this sub-section we replace $U_{M t}$ with the sub-index of macro uncertainty that fluctuates only as a result of the uncertainty in the 73 real activity variables of the macro dataset $\mathcal{X}^{M}$. The $h=1$ month-ahead uncertainty in real activity variables is denoted $U_{R t}$. We study the system $\mathbf{X}_{t}^{R}=\left(U_{R t}, i p_{t}, U_{F t}\right)^{\prime}$ using the same identifying restrictions applied above to the system with $U_{M t}$.

This system is interesting because it puts the spotlight on real activity uncertainty as the source of the large macro uncertainty shock found in the unconstrained set in 1970:12. We search (now for the $\mathbf{X}_{t}^{R}$ system) across all observations in the unconstrained set $\hat{\mathcal{B}}$ for the month in which the shock to $U_{R t}$, denoted $e_{R}(\mathbf{B})$, is largest. Doing so for each of the 1.5 million rotations, we find that the date with the greatest number of maxima across all 1.5 million rotations (27\% of them) is 1970:12, while the date with the second greatest is the month of the Lehman collapse, 2008:09.

The left panel of Figure 8 displays the time series plot of $U_{R t}$, which unlike $U_{M t}$, exhibits a big spike upward in 1970:12. The right panel shows the identified set of IRFs for this system when the 75th-percentile value of $e_{R t}(\mathbf{B})$ in $\hat{\mathcal{B}}$ is again used to parameterize a large shock. The 75th-percentile value is equal to 4.7 in 1970:12 and 4.9 in 2008:09. Although this value is smaller in 1970:12 than in 2008:08, the former episode has more extreme large shocks than latter, which explains why it is the episode with more maxima in the unconstrained set. (The 
99th-percentile value for $e_{R t}(\mathbf{B})$ is 6.6 in 1970:12 compared to 6.3 in 2008:09.) The IRFs are qualitatively similar to the base case system that uses $U_{M t}$, with positive financial uncertainty shocks driving down production sharply and persistently, real (but not financial) uncertainty rising sharply in response to a negative first moment shock to production, and positive real uncertainty shocks driving up production, rather than down. The main difference from the system with $U_{M t}$ is that production now rises more sharply and more persistently in response to a positive real uncertainty shock than it did to a macro uncertainty shock.

\subsection{Recursive Identification and Longer Horizon Uncertainty}

The assumptions in our event and correlation constraints do not rule out the possibility of a recursive structure. If such a structure is consistent with the data, our identifying restrictions are free to recover it. With three variables in the SVAR, there are six possible recursive orderings corresponding to six different $3 \times 1$ vectors of elements of $\mathbf{B}$ that must be jointly zero. It is straightforward to assess whether our identified solutions are consistent with a recursive structure by examining the distribution of solutions in the constrained set for four elements of the B matrix: $\hat{B}_{Y F}, \hat{B}_{Y M}, \hat{B}_{M Y}$, and $\hat{B}_{F M}$. None of the distributions contain any values near zero. The minimum absolute values in the identified for each are 0.002, 0.004, 0.007, and 0.005, respectively. These correspond to the smallest (in absolute terms) impact responses in the identified set reported in Figure 3, subplots $(3,2),(1,2),(2,1)$ and $(1,3)$, respectively. For each case, the $95 \%$ confidence interval for the identified set of impact responses also dose not include zero, showing that, although small in absolute terms, the responses differ significantly from zero once we take into account sampling variability in the data. The implication is that the recursive structure is inconsistent with any recursive ordering across all solutions in the identified set. The finding underscores the challenges of relying on convenient timing assumptions to sort out cause and effect in the relationship between uncertainty and real activity.

The dynamic responses reported for the base case are similar to an SVAR that imposes $B_{F Y}$ and $B_{M F}$ to be zero, or close to zero, while restricting $B_{Y F}$ to be in the range $(-0.02,0)$. If, in addition, either $B_{Y M}$ (giving the impact response of $Y$ to an $e_{M}$ shock) or $B_{Y F}$ (giving the impact response of $Y$ to an $e_{F}$ shock) were estimated to be zero under our constraints, our estimates would recover a recursive structure. But our event and external variable restrictions rule this out. The left panel of Figure 7 above showed why: the event constraints restrict $B_{Y F}$ to be negative and $B_{Y M}$ to be positive. The external variable constraints restrict the magnitude of these parameters.

Finally, we examine longer horizon uncertainty. Figure 9 presents the IRFs when we use a system with $h=12$ month-ahead macro and financial uncertainty, along with $i p_{t}$. This system is denoted $\mathbf{X}_{t}^{(12)}=\left(U_{M t}(12) \text {, } i p_{t}, U_{F t}(12)\right)^{\prime}$, where $U_{M t}(12)$ denotes twelve-month-ahead macro 
uncertainty, and likewise for $U_{F t}(12)$. The same identifying restrictions are used as for the $h=1$ month-ahead base case system. These results are similar to those for the base case that uses $h=1$ month ahead uncertainty.

\section{Conclusion}

A growing body of research establishes uncertainty as a feature of deep recessions but leaves open two key questions: is uncertainty primarily a source of business cycle fluctuations or an endogenous response to them? And does the type of uncertainty matter? The objective of this paper is to address both questions econometrically using small-scale structural VARs capable of nesting a range of theoretical possibilities.

The macro literature on uncertainty has primarily focused on real activity induced macro uncertainty as a driver of economic fluctuations. Using a novel identification approach that imposes economic assumptions on the behavior of the shocks, we find from a variety of parameterizations and specifications that macro uncertainty rises endogenously in response to real activity shocks, contributing to strongly its countercyclical behavior. It is shocks to financial uncertainty, rather than macro uncertainty, that are found to be a driver of economic fluctuations. But macro uncertainty is found to substantially amplify downturns caused by other shocks, indicating that it may magnify recessions even if it doesn't cause them. An implication of these findings is that dynamic equilibrium models should allow for broad-based macro uncertainty to respond endogenously to a variety of shocks, while entertaining the notion that occasional large shocks to uncertainty originating in financial markets may be a source of deep recessions.

Our findings call for a need to better understand how uncertainty in financial markets is transmitted to the macroeconomy, and why the two types of uncertainty have a distinct relationship with economic activity. A burgeoning business cycle literature has begun to postulate theoretical linkages between financial market uncertainty, real/macro uncertainty, and real activity. ${ }^{19}$ Although these models are currently too stylized to be confronted with actual data, they appear capable of generating implications that are consistent at least qualitatively with our finding that positive shocks to financial uncertainty are a driving force of declines in productive activity, while real uncertainty responds endogenously to first moment shocks to productive activity.

\footnotetext{
${ }^{19}$ For example. Benhabib, Liu, and Wang (2017) studies self-fulfilling surges in financial and real uncertainty in a model of informational interdependence and mutual learning; Adrian and Boyarchenko (2012), Brunnermeier and Sannikov (2014), and He and Krishnamurthy (2012) study production economies with financial intermediaries that give rise to time-varying GDP vulnerabilities (downside real risk) as a function of time-varying financial frictions; hence financial uncertainty drives both GDP and its volatility.
} 
Acknowledgements Ludvigson acknowledges financial support from the C.V. Starr Center for Applied Economics at NYU. Ng acknowledges support from the National Science Foundation under grant SES-0962431 and SES-1558623. We thank seminar participants at the 2016 ME, EFFE, and EF\&G group meetings at the NBER, the CEPR-DRG-SAFE Conference on Banking, Monetary Policy, and Macroeconomic Performance in Frankfurt May 2016, the New Developments in Business Cycle Analysis conference in Rome June 2016, the 2016 Econometric Society meetings, the Joint Central Bankers Conference November 2017, the T2M Macro conference in Paris March 2018, the FRBNY and BOG Developments in Empirical Macroeconomics Conference May 10-11, 2018, Collegio Carlo Alberto, the Federal Reserve Board, the Federal Reserve Bank of Philadelphia, HEC Montreal, Johns Hopkins Carey School, MIT Sloan, NYU, SSE Swedish House of Finance, the University of Zurich/Swiss Finance Institute, UC Davis, UNC Kenan-Flagler, Washington University in St. Louis, and Yale for helpful comments. We are grateful to Ian Dew-Becker, Laurent Ferrara, John Leahy, Kurt Lunsford, Jóse Luis Montiel Olea, Mikkel Plagborg-Møller, Jim Stock, and Stephen Terry for many valuable discussions.

\section{References}

Abel, A. B. (1983): "Optimal Investment under Uncertainty," American Economic Review, $73(1), 228-233$.

Adrian, T., and N. Boyarchenko (2012): "Intermediary leverage cycles and financial stability," Unpublished paper, Federal Reserve Bank of New York.

Ai, H., K. Li, And F. YAng (2015): "Financial Intermediation and Capital Misallocation," Unpublished manuscript, University of Minnesota.

Andrews, D. W. K., and G. Soares (2010): "Inference for parameters defined by moment inequalities using generalized moment selection," Econometrica, 78(1), 119-157.

Angelini, G., E. Bacchiocchi, G. Caggiano, and L. Fanelli (2018): "Uncertainty across volatility regimes," Journal of Applied Econometrics.

Antolín-Díaz, J., and J. F. Rubio-Ramírez (2018): "Narrative sign restrictions for SVARs," American Economic Review, 108(10), 2802-29.

Arellano, C., Y. Bai, and P. Kehoe (2011): "Financial Markets and Fluctuations in Uncertainty," Federal Reserve Bank of Minneapolis Research Department Staff Report 466.

Bachmann, R., S. Elstner, and E. R. Sims (2013): "Uncertainty and Economic Activity: Evidence from Business Survey Data," American Economic Journal: Macroeconomics, 5(2), $217-49$.

Bachmann, R., and G. Moscarini (2011): "Business Cycles and Endogenous Uncertainty," Discussion paper, Society for Economic Dynamics.

Baker, S. R., And N. Bloom (2013): "Does Uncertainty Reduce Growth? Using Disasters as Natural Experiments," National Bureau of Economic Research Working Paper 19475.

Baker, S. R., N. Bloom, and S. J. Davis (2016): "Measuring economic policy uncertainty," The Quarterly Journal of Economics, 131(4), 1593-1636. 
Bar-Ilan, A., And W. C. Strange (1996): "Investment lags," The American Economic Review, 86(3), 610-622.

BASU, S., AND B. Bundick (2017): "Uncertainty shocks in a model of effective demand," Econometrica, 85(3), 937-958.

Baumeister, C., And J. D. Hamilton (2015): "Sign restrictions, structural vector autoregressions, and useful prior information," Econometrica, 83(5), 1963-1999.

Bekaert, G., M. Hoerova, and M. L. Duca (2013): "Risk, Uncertainty and Monetary Policy," Journal of Monetary Economics, 60(7), 771-88.

Benhabib, J., X. Liu, and P. Wang (2017): "Financial Markets, the Real Economy, and Self-Fulfilling Uncertainties," Unpublished manuscript, NYU.

Berger, D., I. Dew-Becker, and S. Giglio (2016): "Contractionary volatility or volatile contractions?," Unpublished paper, Northwestern University.

Bernanke, B. S. (1983): "Irreversibility, Uncertainty, and Cyclical Investment," The Quarterly Journal of Economics, 98(1), 85-106.

Bloom, N. (2009): "The Impact of Uncertainty Shocks," Econometrica, 77(3), 623-85. $153-175$

(2014): "Fluctuations in Uncertainty," The Journal of Economic Perspectives, pp.

Bloom, N., M. Floetotto, N. Jaimovich, I. Saporta-Eksten, and S. J. Terry (2018): "Really uncertain business cycles," Econometrica, 86(3), 1031-1065.

Bollerslev, T., G. Tauchen, and H. Zhou (2009): "Expected stock returns and variance risk premia," Review of Financial Studies, 22(11), 4463-4492.

Brunnermeier, M., And Y. SAnnikov (2014): "A Macroeconomic Model with a Financial Sector," American Economic Review, 104, 379-421.

Campbell, J. Y., S. Giglio, C. Polk, and R. Turley (2018): "An intertemporal CAPM with stochastic volatility," Journal of Financial Economics, 128(2), 207-233.

Campbell, J. Y., and L. Hentschel (1992): "An Asymmetric Model of Changing Volatility in Stock Returns," Journal of Financial Economics, 31, 281-318.

Carriero, A., T. E. Clark, and M. Marcellino (2018): "Endogenous Uncertainty," Federal Reserve Bank of Cleveland Working Papers, (WP 18-05).

EngLE, R. F., AND V. K. NG (1993): "Measuring and testing the impact of news on volatility," The journal of finance, 48(5), 1749-1778.

Fajgelbaum, P. D., E. Schaal, and M. Taschereau-Dumouchel (2017): "Uncertainty traps," The Quarterly Journal of Economics, 132(4), 1641-1692.

Favilukis, J., S. C. Ludvigson, and S. Van Nieuwerburgh (2017): "The Macroeconomic Effects of Housing Wealth, Housing Finance and Limited Risk Sharing in General Equilibrium," Journal of Political Economy, 125(1), 140-223.

Fernández-Villaverde, J., J. F. R.-R. Pablo Guerrón-Quintana, and M. Uribe (2011): "Risk Matters: The Real Effects of Volatility Shocks," American Economic Review, $6(101), 2530-61$. 
Fostel, A., And J. Geanakoplos (2012): "Why does bad news increase volatility and decrease leverage?," Journal of Economic Theory, 147(2), 501-525.

French, K., G. W. Schwert, and R. F. Stambaugh (1987): "Expected Stock Returns and Volatility," Journal of Financial Economics, 19, 3-30.

Gafarov, B., M. Meier, and J. M. Olea (2015): "Projection Inference for Set-Identified SVARs," Unpublished paper, New York University.

Gertler, M., And P. KARAdi (2015): "Monetary policy surprises, credit costs, and economic activity," American Economic Journal: Macroeconomics, 7(1), 44-76.

Gilchrist, S., J. W. Sim, and E. Zakrajsek (2010): "Uncertainty, Financial Frictions, and Investment Dynamics," Society for Economic Dynamics 2010 Meeting Papers No.1285.

Glaeser, E. L., T. Santos, and E. G. Weyl (2017): After the Flood: How the Great Recession Changed Economic Thought. University of Chicago Press.

Gomes, J. F., And L. Schmid (2016): "Equilibrium Asset Pricing with Leverage and Default," http://finance.wharton.upenn.edu/gomesj/Research/CreditMarkets.pdf.

Hartman, R. (1972): "The effects of price and cost uncertainty on investment," Journal of economic theory, 5(2), 258-266.

He, Z., And A. Krishnamurthy (2012): "A Model of Capital and Crises," Review of Economic Studies, 79, 735-777.

Ilut, C. L., And H. SAijo (2016): "Learning, confidence, and business cycles," Discussion paper, National Bureau of Economic Research.

Jurado, K., S. C. Ludvigson, and S. Ng (2015): "Measuring Uncertainty," The American Economic Review, 105(3), 117-1216.

Kilian, L., And H. Lutkepohl (2016): Structural Vector Autoregressive Analysis. Cambridge University Press.

Kraft, H., E. Schwartz, And F. Weiss (2018): "Growth options and firm valuation," European Financial Management, 24(2), 209-238.

LEDUC, S., AND Z. LiU (2016): "Uncertainty shocks are aggregate demand shocks," Journal of Monetary Economics, 82, 20-35.

Lettau, M., S. Ludvigson, and C. Steindel (2002): "Monetary policy transmission through the consumption-wealth channel," FRBNY Economic Policy Review, 5, 117-133.

Lintner, J. (1965): "Security Prices, Risk and Maximal Gains from Diversification," Journal of Finance, 20, 587-615.

Ludvigson, S. C., S. Ma, And S. NG (2016): "Shock Restricted Structural VectorAutoregressions," Manuscript, New York University.

Ludvigson, S. C., And S. NG (2007): "The Empirical Risk-Return Relation: A Factor Analysis Approach," Journal of Financial Economics, 83(1), 171-222.

McCracken, M. W., And S. NG (2016): "FRED-MD: A monthly database for macroeconomic research," Journal of Business 8 Economic Statistics, 34(4), 574-589.

McDonald, R., And D. Siegel (1986): "The Value of Waiting to Invest," The Quarterly Journal of Economics, 101(4), 707-728. 
Mertens, K., And M. O. Ravn (2013): "The dynamic effects of personal and corporate income tax changes in the United States," The American Economic Review, 103(4), 12121247.

(2014): "A reconciliation of svar and narrative estimates of tax multipliers," Journal of Monetary Economics, 68, S1-S19.

Moon, H. R., And F. Schorfheide (2012): "Bayesian and frequentist inference in partially identified models," Econometrica, 80(2), 755-782.

Moon, H. R., F. Schorfheide, and E. Granziera (2013): "Inference for VARs Identified with Sign Restrictions," Unpublished Paper.

Nakamura, E., D. Sergeyev, and J. Steinsson (2017): "Growth-rate and uncertainty shocks in consumption: Cross-country evidence," American Economic Journal: Macroeconomics, 9(1), 1-39.

Nelson, D. B. (1991): "Conditional Heteroskedasticity in Asset Returns: A New Approach," Econometrica, 59(2), 347-70.

NG, S., And J. H. Wright (2013): "Facts and Challenges from the Great Recession for Forecasting and Macroeconomic Modeling," Journal of Economic Literature, 51(4), 11201154.

OI, W. Y. (1961): "The desirability of price instability under perfect competition," Econometrica: journal of the Econometric Society, pp. 58-64.

Pastor, L., And P. Veronesi (2006): "Was There A Nasdaq Bubble in the Late 1990s?," Journal of Financial Economics, 81, 61-100.

PÁstor, L., And P. Veronesi (2013): "Political uncertainty and risk premia," Journal of Financial Economics, 110(3), 520-545.

Piffer, M., and M. Podstawski (2017): "Identifying uncertainty shocks using the price of gold," The Economic Journal, 128(616), 3266-3284.

RAmey, V. A. (2016): "Macroeconomic shocks and their propagation," in Handbook of Macroeconomics, vol. 2, pp. 71-162. Elsevier.

Rubio Ramírez, J. F., D. F. Waggoner, and T. Zha (2010): "Structural vector autoregressions: Theory of identification and algorithms for inference," The Review of Economic Studies, 77(2), 665-696.

Schwert, G. W. (1989): "Why Does Stock Market Volatility Change over Time," Journal of Finance, 44(5), 1115-53.

Segal, G., I. Shaliastovich, and A. Yaron (2015): "Good and bad uncertainty: Macroeconomic and financial market implications," Journal of Financial Economics, 117(2), 369397.

Sharpe, W. (1964): "Capital Asset Prices: A Theory of Market Equilibrium under Conditions of Risk," Journal of Finance, 19, 425-444.

Stock, J. H., and M. W. Watson (2008): "NBER Summer Institute Minicourse 2008: What's New in Econometrics: Time Series, Lecture 7: Structural VARs," Cambridge, Mass.: National Institute for Economic Research. www. nber. org/minicourse_2008. html.

(2012): "Disentangling the Channels of the 2007-09 Recession," Brookings Papers on Economic Activity, p. 81. 
VAn Nieuwerburgh, S., And L. VeldkAmp (2006): "Learning asymmetries in real business cycles," Journal of monetary Economics, 53(4), 753-772.

Whaley, R. E. (2000): "The investor fear gauge," The Journal of Portfolio Management, $26(3), 12-17$. 
Figure 1: Macro and Financial Uncertainty Over Time

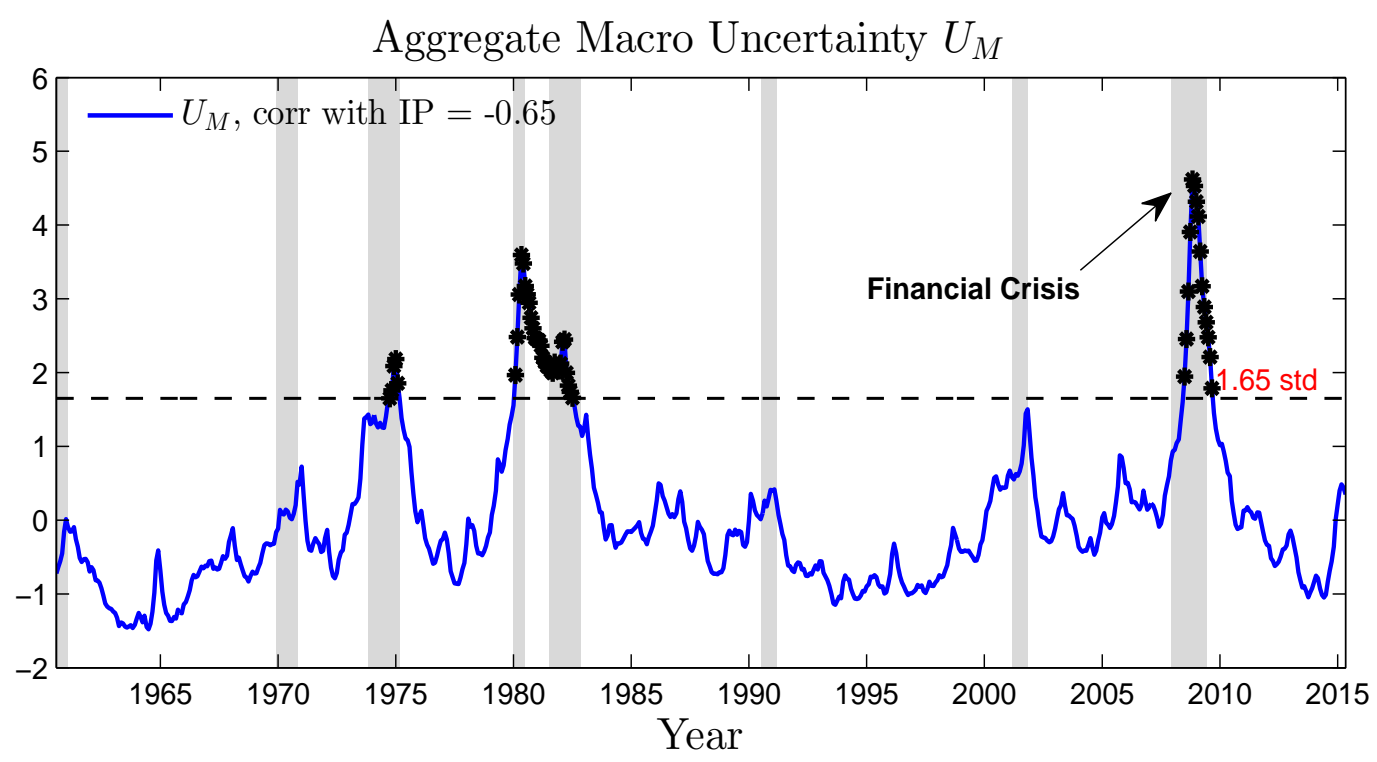

Aggregate Financial Uncertainty $U_{F}$

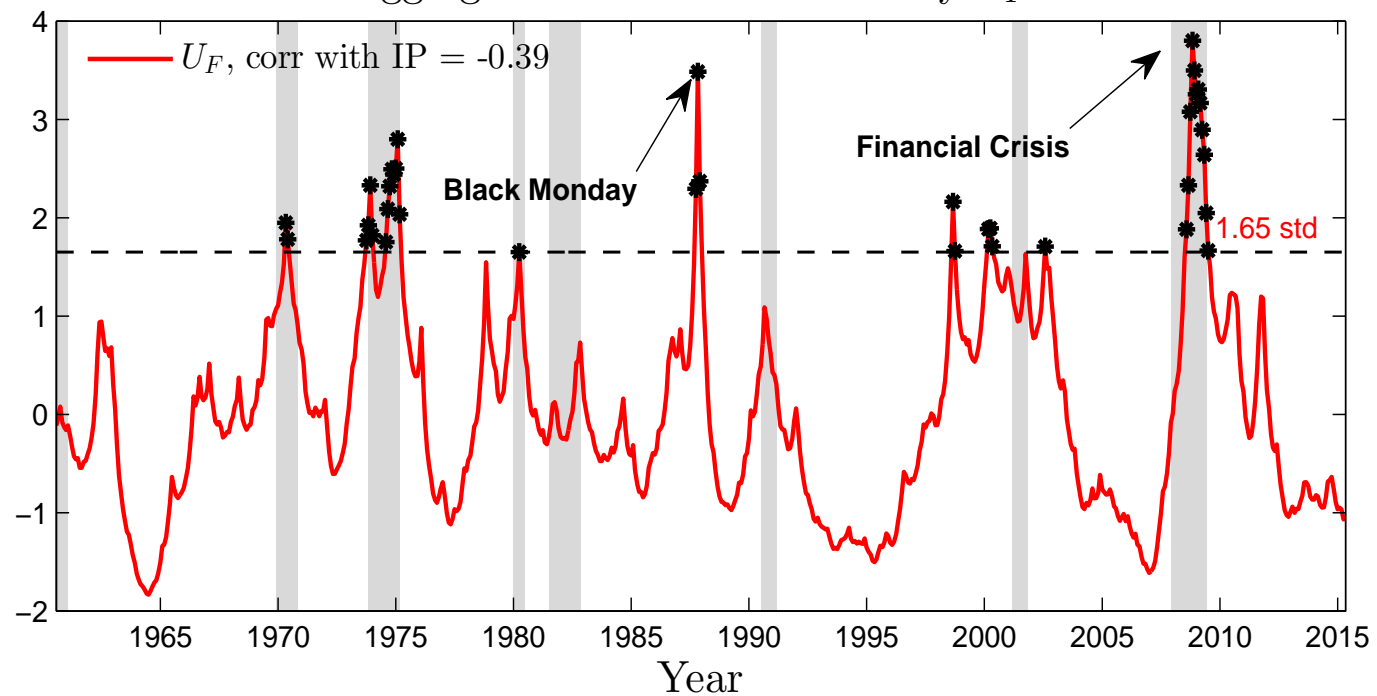

The panels plot the time series of macro uncertainty $U_{M}$ and financial uncertainty $U_{F}$ expressed in standardized units. Shaded areas correspond to NBER recession dates. The horizontal line corresponds to 1.65 standard deviations above the unconditional mean of each series (which has been normalized to zero); the black dots are months when uncertainty is at least 1.65 standard deviations above the mean. Correlations with the 12-month moving average of IP growth are reported. The data span the period 1960:07 to 2015:04. 
Figure 2: Structural Shocks $e$

Time Series of e Shock from Base Case

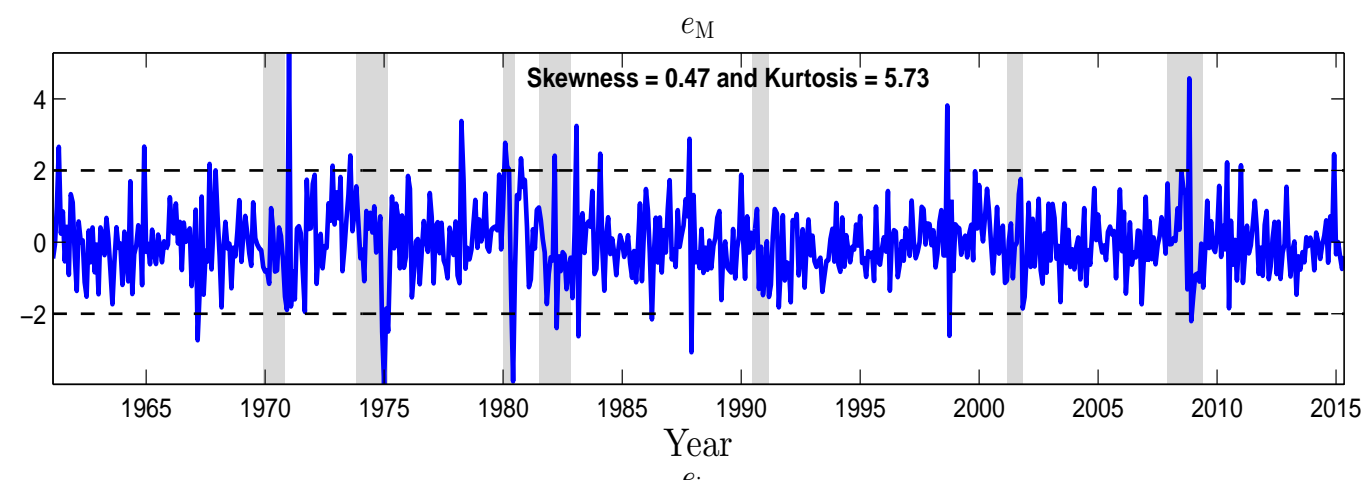

$e_{\mathrm{ip}}$

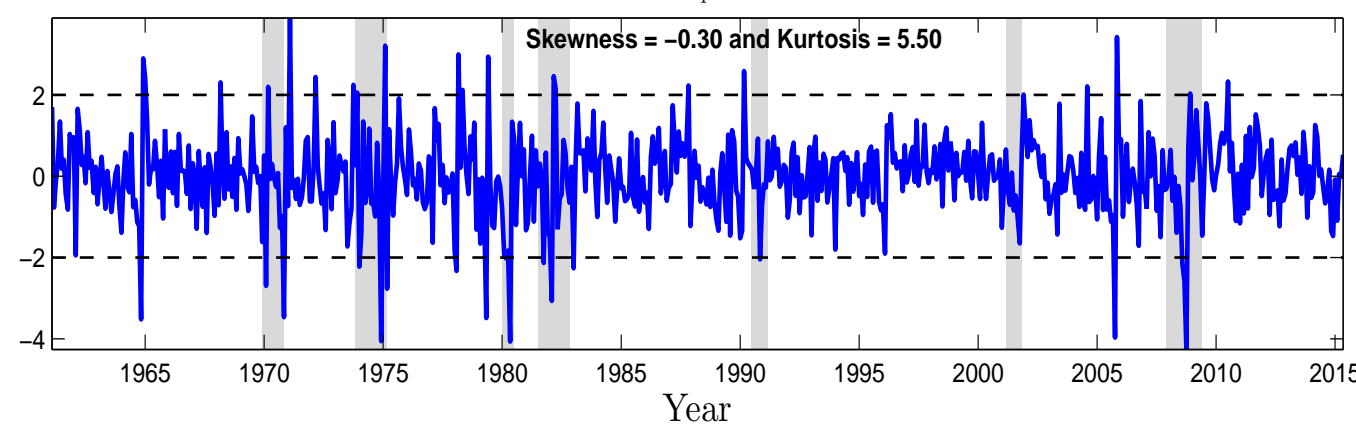

$e_{\mathrm{F}}$

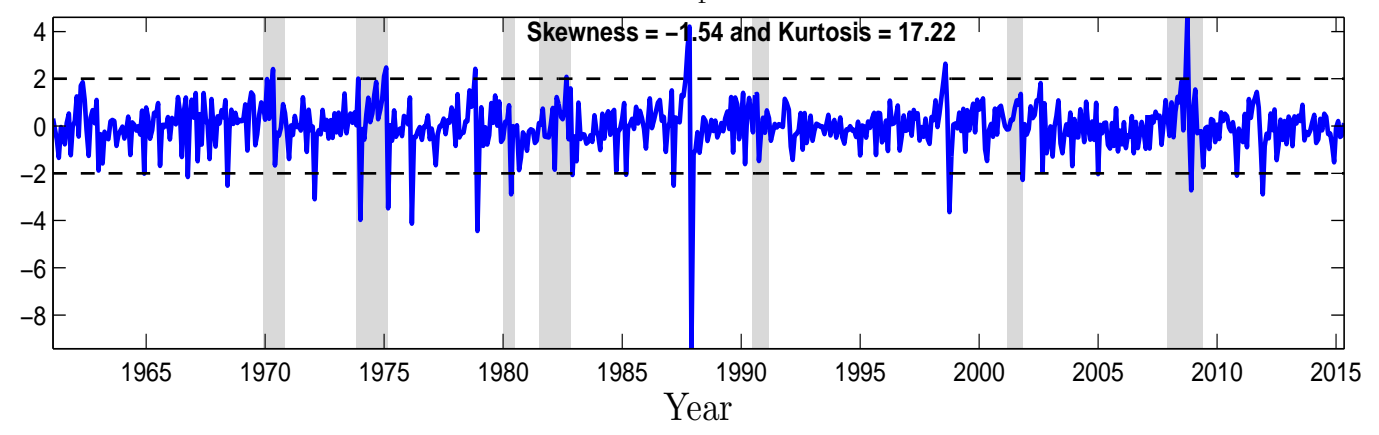

Large Shocks

Positive $e_{\mathrm{M}}$ exceeding 2 standard deviations

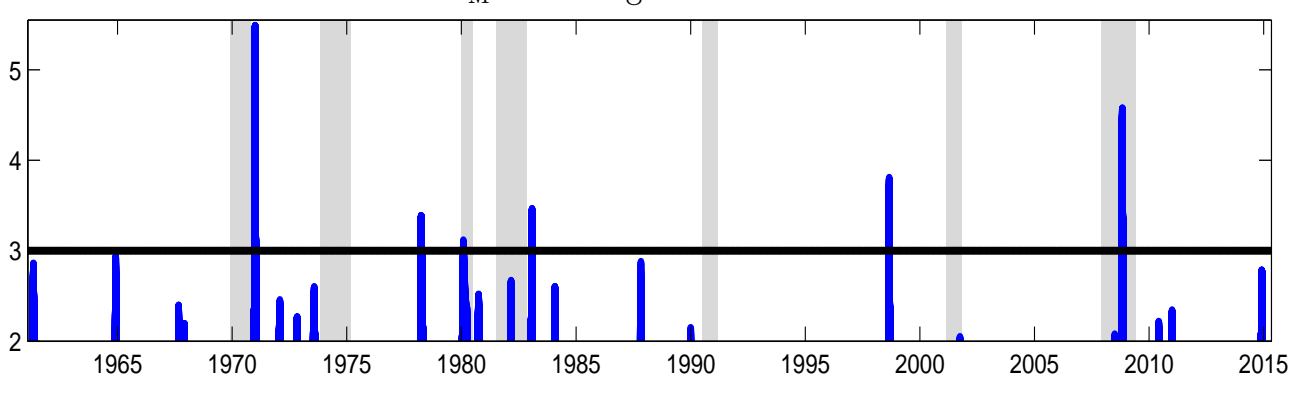

Negative $e_{\text {ip }}$ exceeding 2 standard deviations

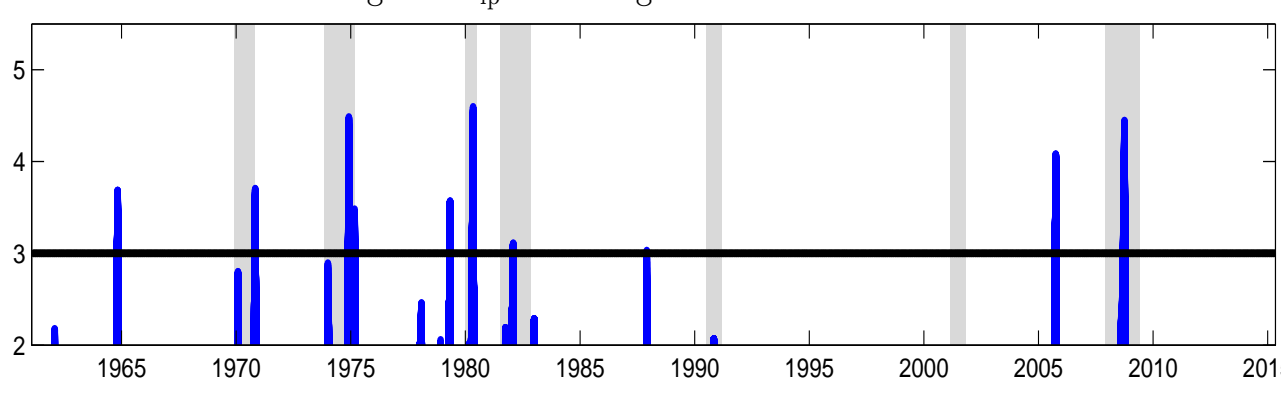

Positive $e_{\mathrm{F}}$ exceeding 2 standard deviations

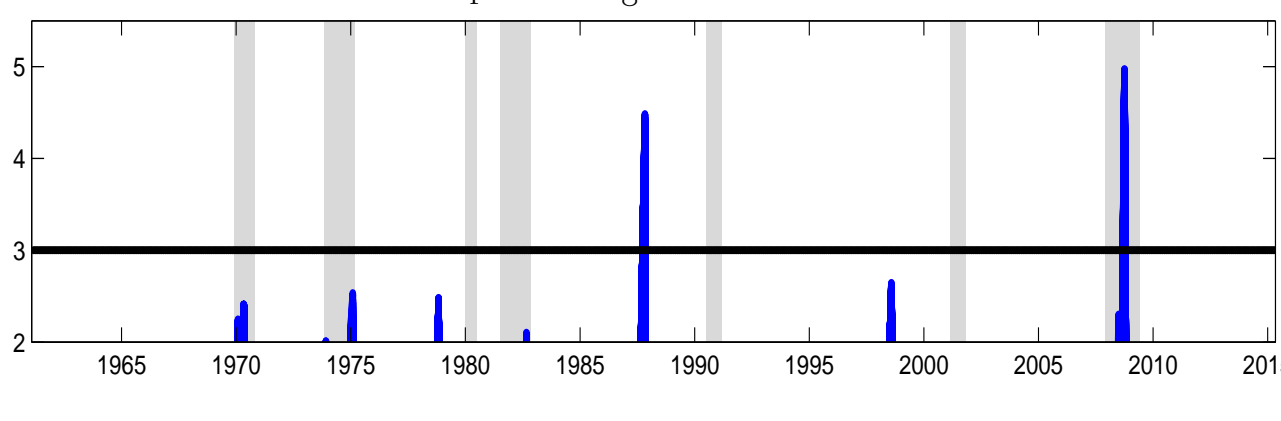

The left panel reports the time series of the structural shocks $e=B^{-1} \eta_{t}$ for maxG solution of the base case. The horizontal line corresponds to 3 standard deviations above/below the unconditional mean of each series. The right panel exhibits all shocks in the identified set that are at least 2 standard deviations above the unconditional mean for $e_{M}$ and $e_{F}$ and at least 2 standard deviations below the mean for $e_{i p}$. The parameters $\bar{k}_{1}, \bar{k}_{2}, \bar{k}_{3}$ and $\bar{k}_{4}$ are at 75th-percentile of the unconstrained set. The sample spans the period 1960:07 to 2015:04. 
Figure 3: SVAR $\left(U_{M}, i p, U_{F}\right)^{\prime}$
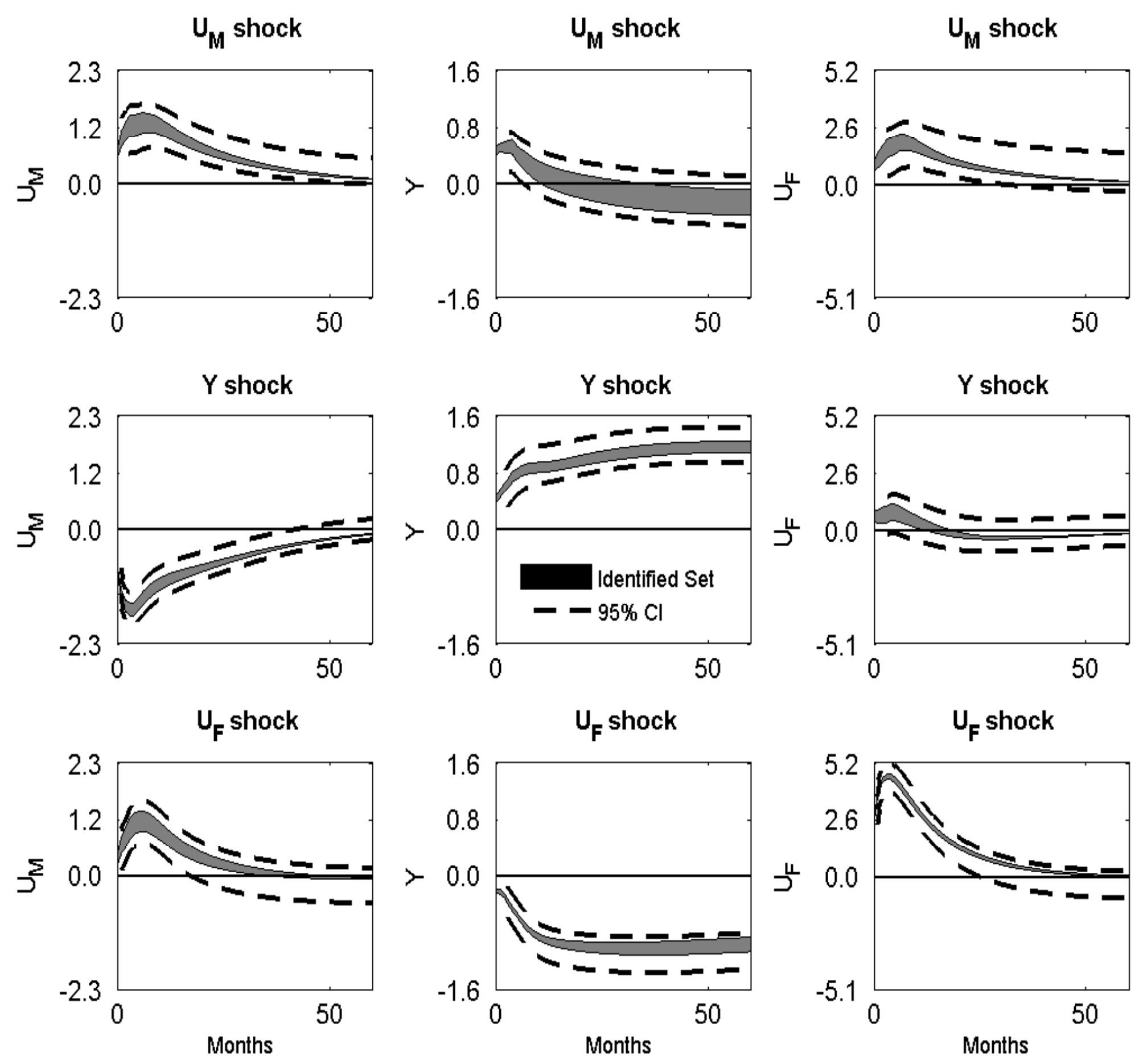

The solid lines report the identified set of impulse response to positive, one standard deviation shocks in units of percentage points for the base case system. The dashed lines report 95 percent confidence intervals (CI) for the identified set. The sample spans the period 1960:07 to 2015:04. 
Figure 4: SVAR $\left(U_{M}, i p, U_{F}\right)^{\prime}:$ Hypothetical $B_{M Y}=0$ and $B_{M F}=0$
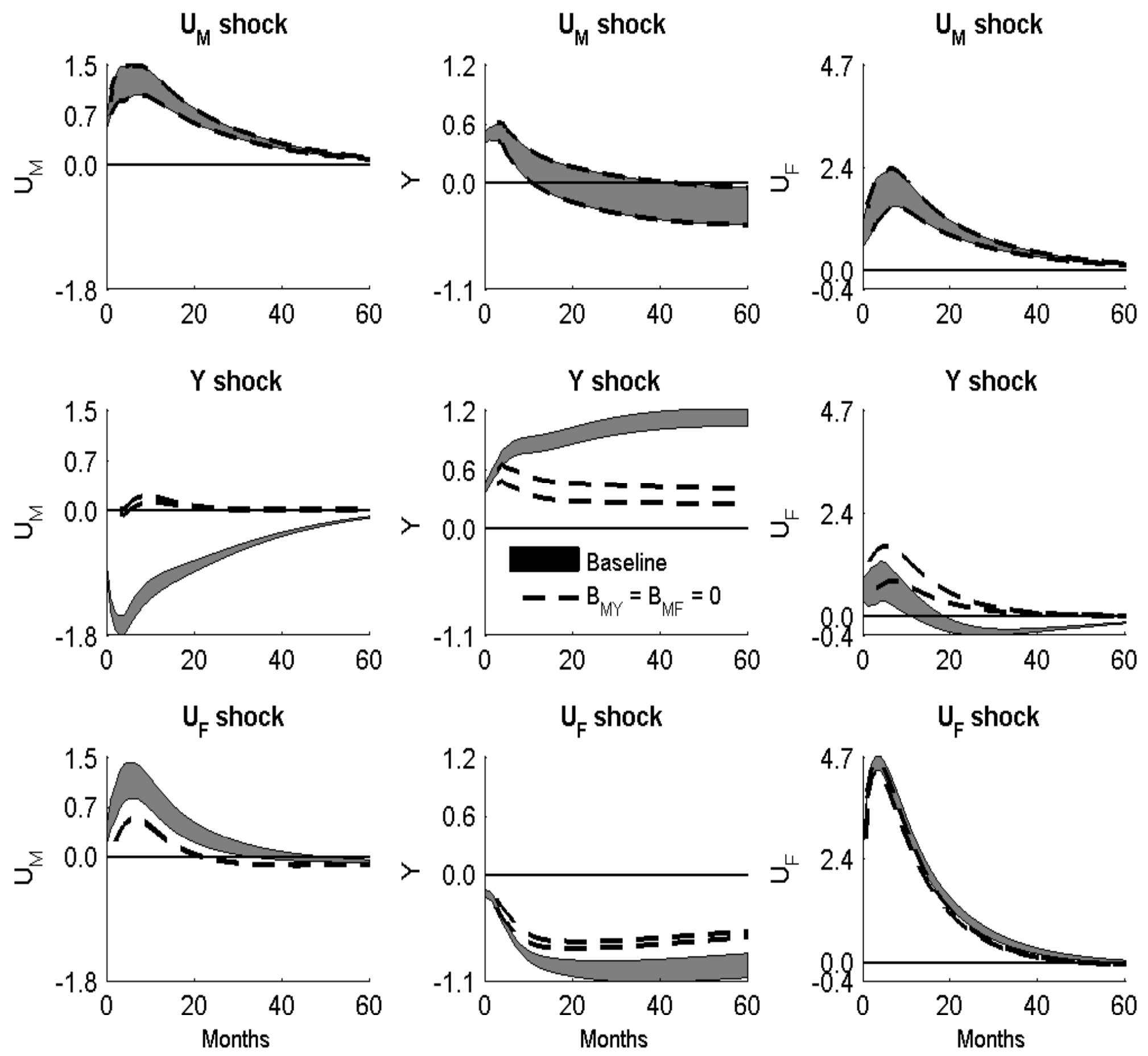

The figure reports the impulse responses of SVAR $\left(U_{M}, i p, U_{F}\right)^{\prime}$ under the hypothetical parameter values $B_{M Y}=0$ and $B_{M F}=0$. The shaded areas report the identified set of impulse response to positive, one standard deviation shocks in units of percentage points for the base case system. The dashed lines report the hypothetical responses. The sample spans the period 1960:07 to 2015:04. 
Figure 5: SVAR $\left(E P U, i p, U_{F}\right)^{\prime}$

\section{EPU Over Time}

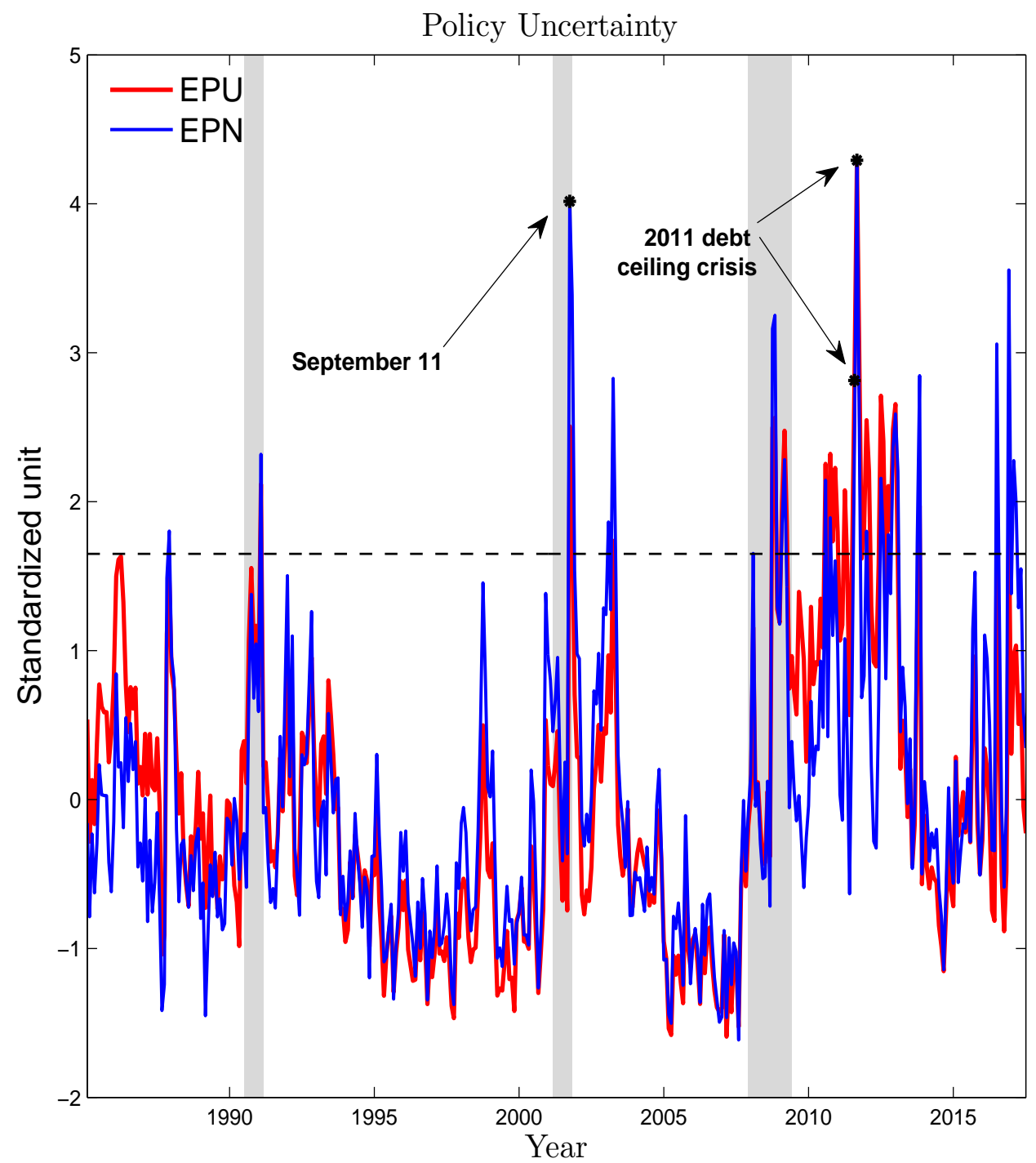

Impulse Response Function
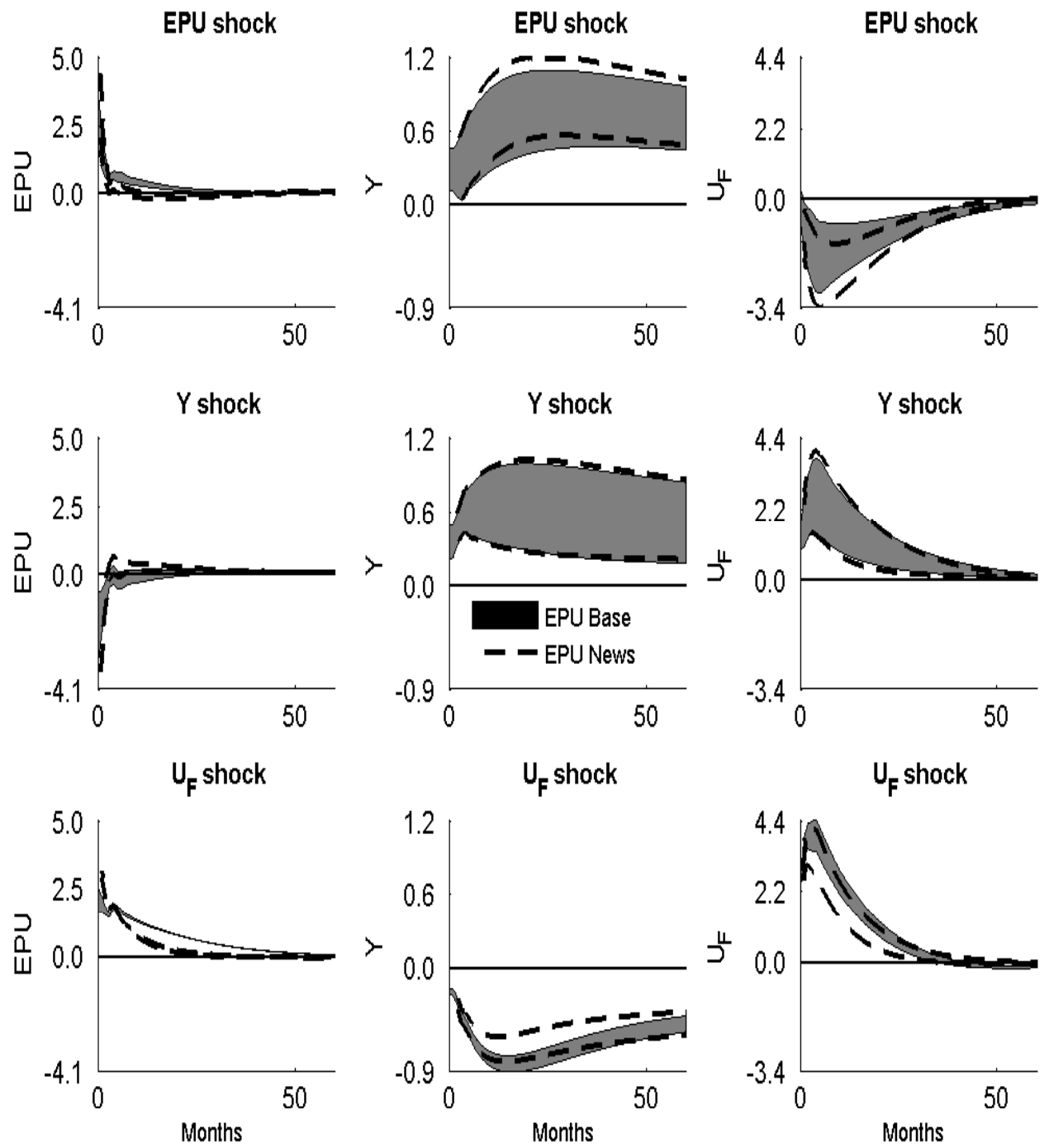

The left panel plots the time series of baseline policy uncertainty $E P U$ and news-based $E P N$, expressed in standardized units. Shaded areas correspond to NBER recession dates. The horizontal line corresponds to 1.65 standard deviations above the unconditional mean. The right panel displays impulse responses to one standard deviation shocks. Response units are reported in percentage points. The parameters $\bar{k}_{1}, \bar{k}_{2}, \bar{k}_{3}$ and $\bar{k}_{4}$ are at 75 th-percentile of the unconstrained set. Additional identifying restriction: for EPU, $e_{E P U, t_{3}} \geq 2$ for for all $t_{3} \in\{2011: 07,2011: 08\}$. The sample spans the period 1987:01 to $2015: 04$. 
Figure 6: IRFs of SVAR $\left(U_{M}, i p, U_{F}\right)^{\prime}$ under Alternative Parameters

Change in 1987 and 1970 Event
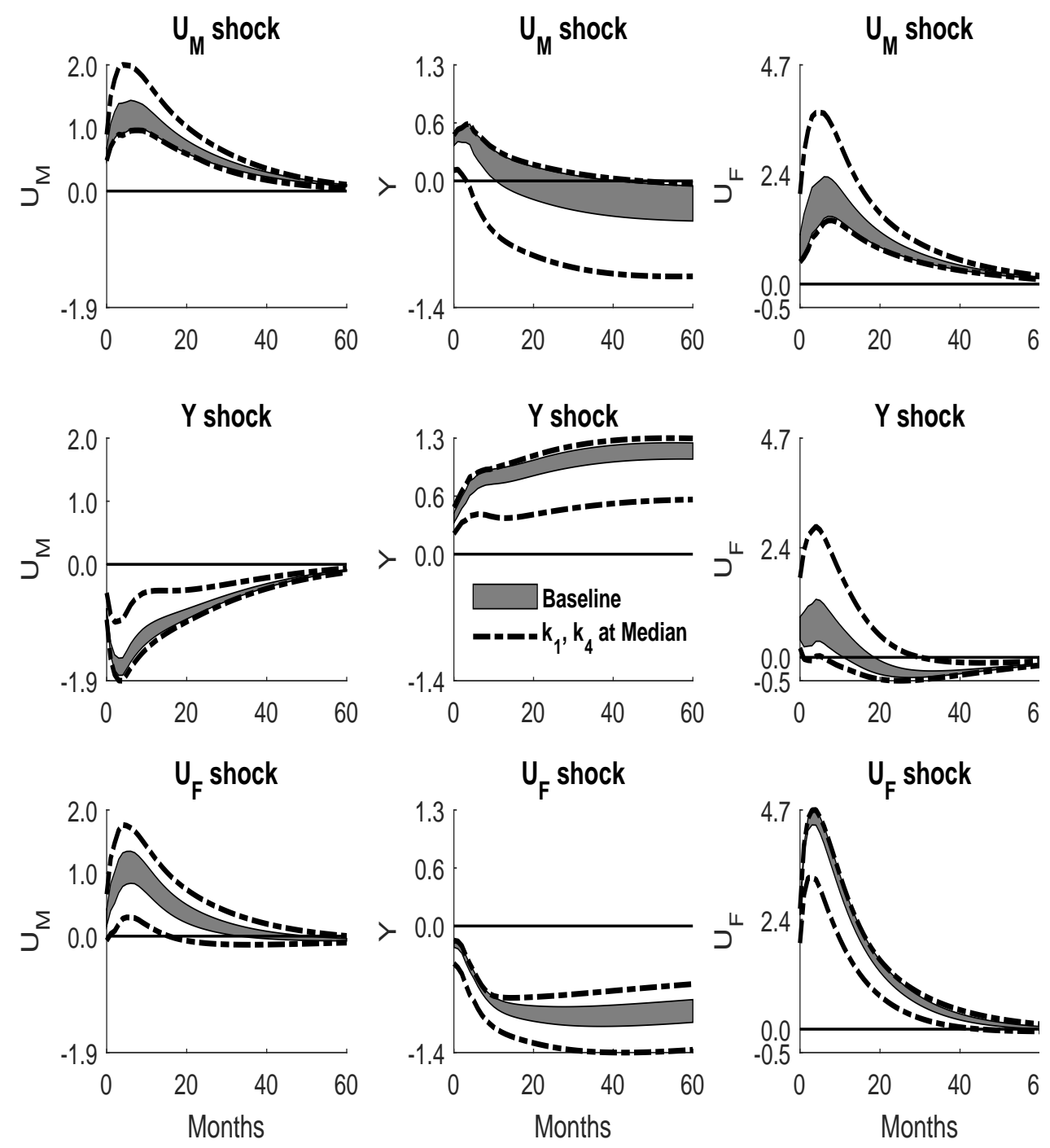

Change in Lehman Event
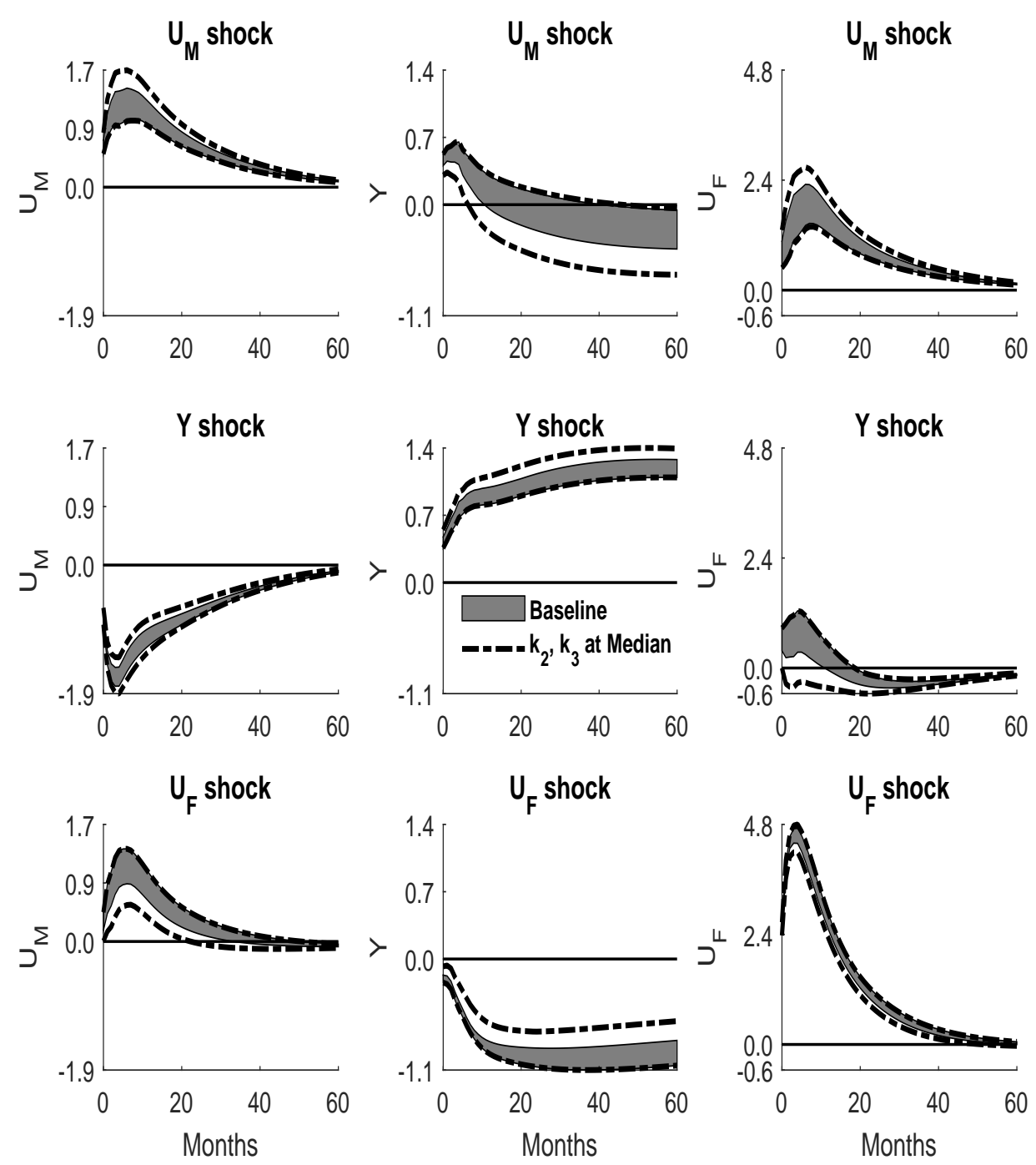

The left panel reports sets of solutions obtained when $\bar{k}_{1}$ and $\bar{k}_{4}$ are slackened to median from 75 th percentile of the unconstrained set while the other parameters are held fixed at their baseline values. The right panel reports sets of solutions obtained when the $\bar{k}_{2}$ and $\bar{k}_{3}$ are slackened to median from 75th-percentile of the unconstrained set while the other parameters are held fixed at their baseline values. The sample spans the period 1960:07 to 2015:04. 
Figure 7: Distribution of $B_{Y F}$ and $B_{Y M}$
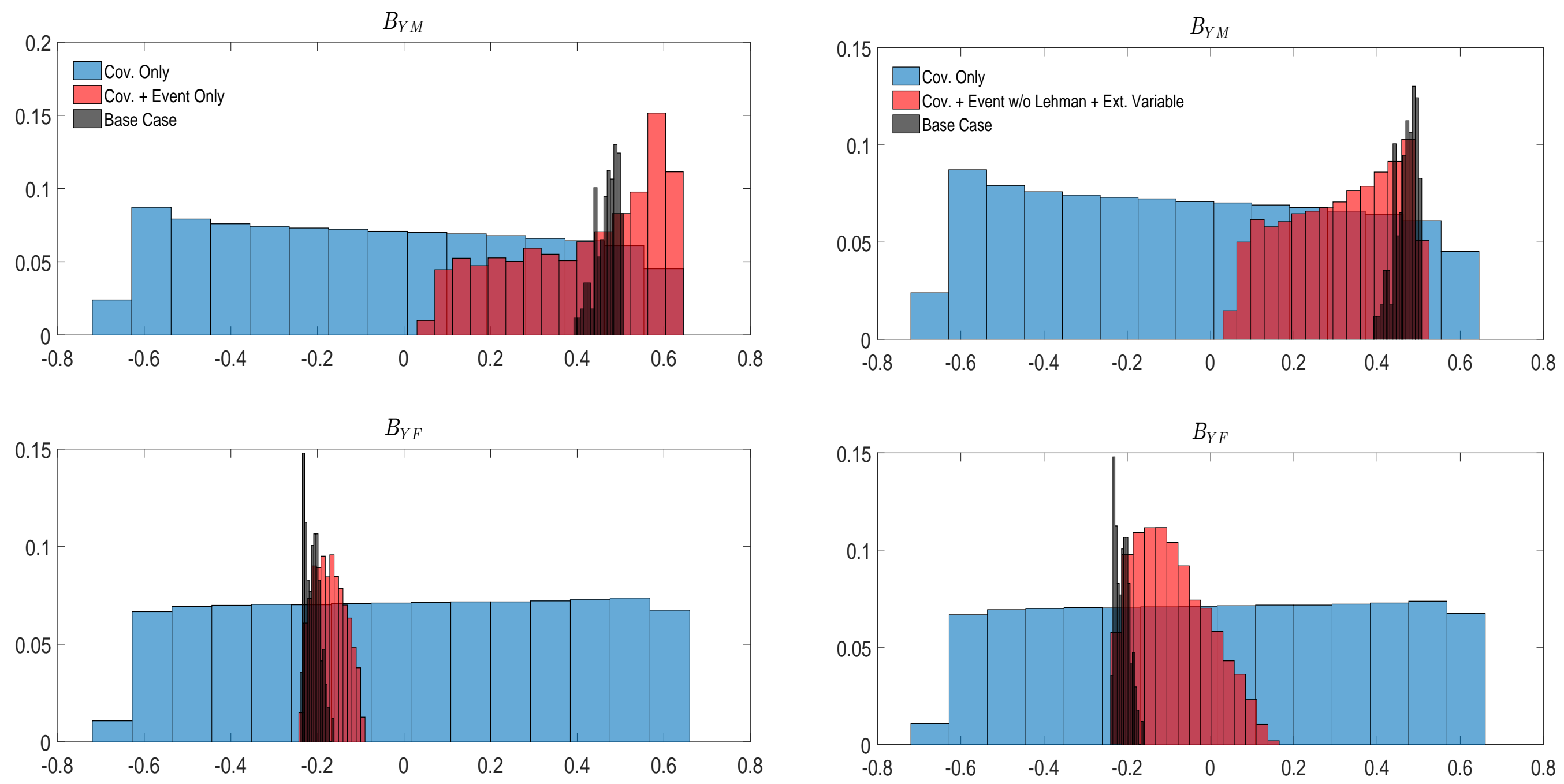

The left panels exhibit the distribution of $B_{Y M}$ (upper panel) and $B_{Y F}$ (lower panel) when only the covariance restrictions are imposed (blue), when only covariance + event constraints are imposed (red) and for the base case where all covariance+event+external variable constraints are imposed (black). The right panels exhibit the distribution of $B_{Y M}$ (upper panel) and $B_{Y F}$ (lower panel) when only the covariance restrictions are imposed (blue), when only covariance + external variable + event constraints without the Lehman event are imposed (red) and for the base case where all covariance+event+external variable constraints are imposed (black). The sample spans the period 1960:07 to 2015:04. 
Figure 8: $\mathbf{S V A R}\left(U_{R}, i p, U_{F}\right)^{\prime}$

\section{Real Uncertainty Over Time}

Real Uncertainty $U_{R}$

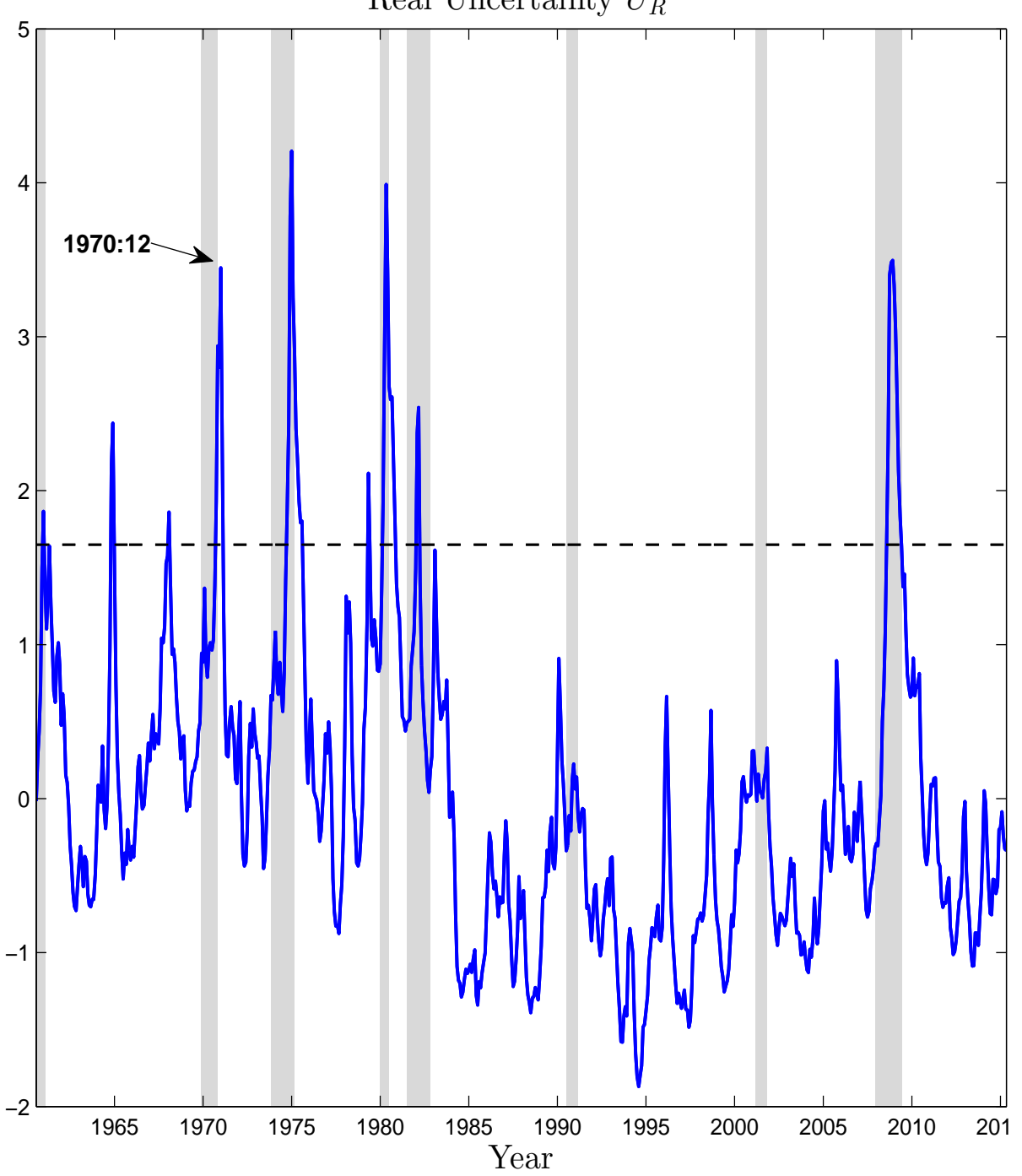

Impulse Response Function
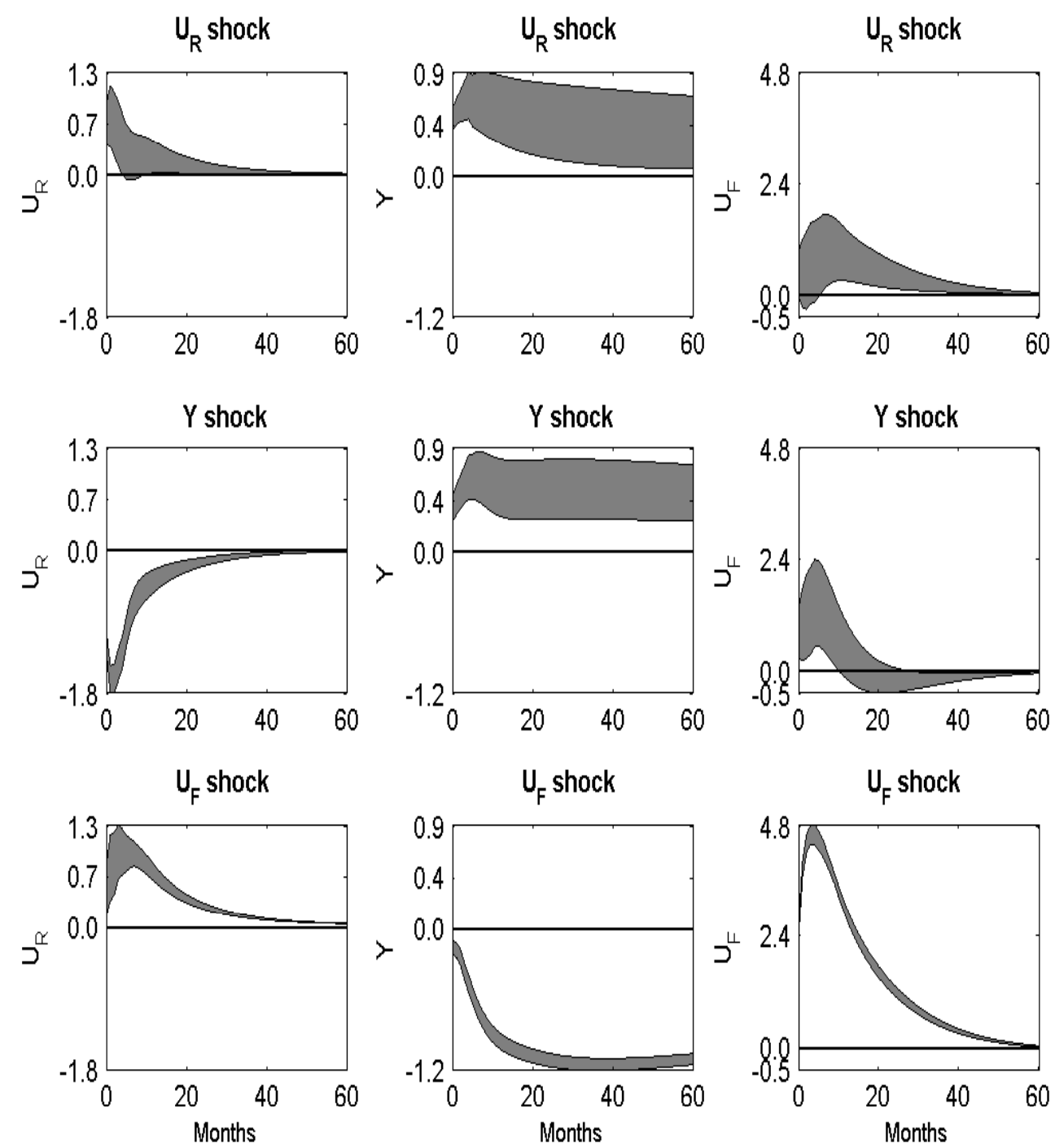

The left panel plots the time series of real uncertainty, expressed in standardized units. Shaded areas correspond to NBER recession dates. The horizontal line corresponds to 1.65 standard deviations above the unconditional mean. The right panel displays impulse responses to one standard deviation shocks. Response units are reported in percentage points. The parameters $\bar{k}_{1}, \bar{k}_{2}, \bar{k}_{3}$ and $\bar{k}_{4}$ are at 75 th-percentile of the unconstrained set. The sample spans the period 1960:07 to 2015:04. 
Figure 9: SVAR $\left(U_{M}(12), i p, U_{F}(12)\right)^{\prime}$
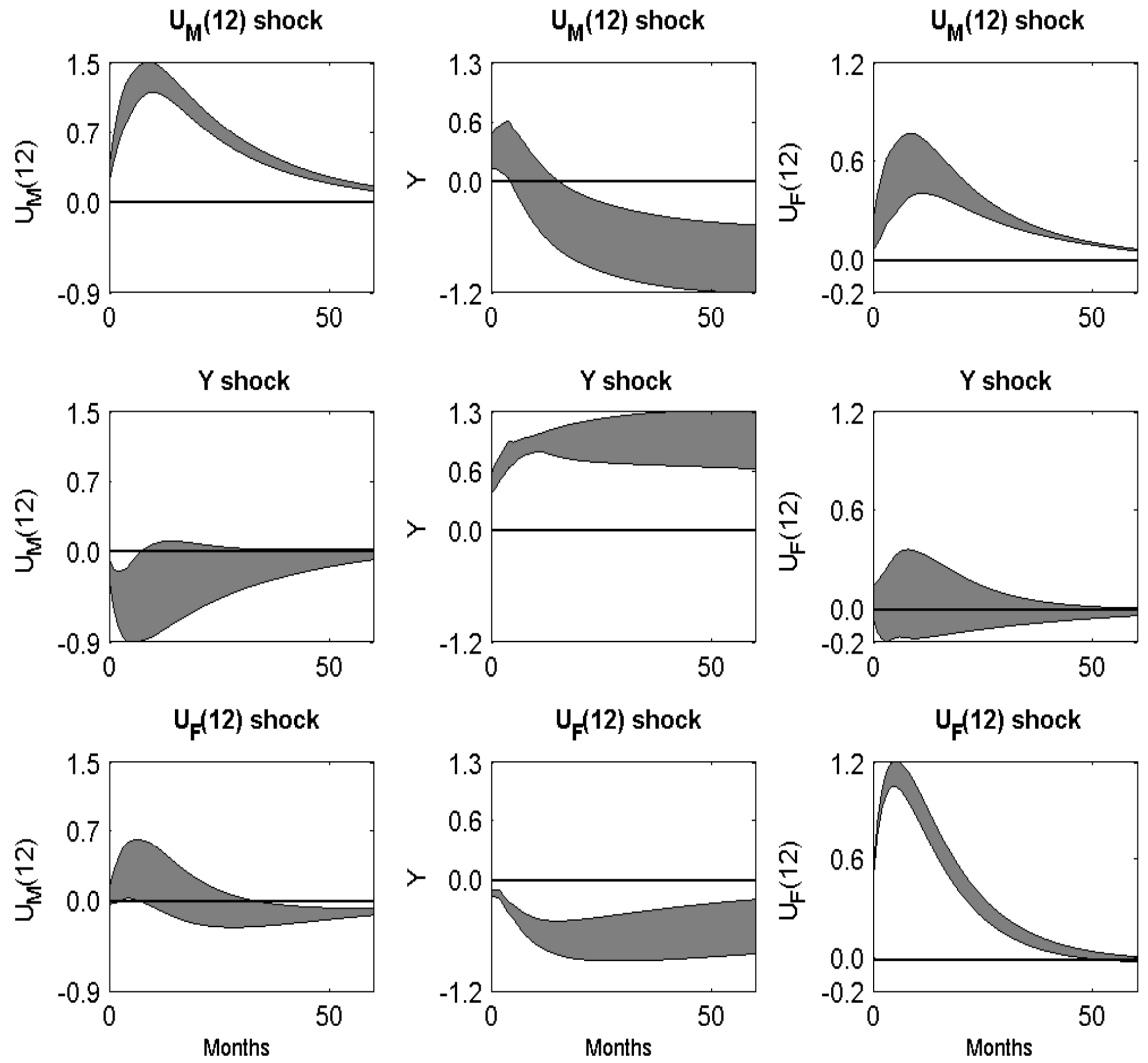

The figure reports the identified set of impulse response to positive, one standard deviation shocks in units of percentage points for the one-year-ahead uncertainties $U_{M}(12)$ and $U_{F}(12)$. The parameters $\bar{k}_{1}, \bar{k}_{2}, \bar{k}_{3}$ and $\bar{k}_{4}$ are at 75th-percentile of the unconstrained set. The sample spans the period 1960:07 to 2015:04. 
Table 1: Variance Decomposition

\begin{tabular}{|c|c|c|c|c|c|c|}
\hline & \multicolumn{3}{|c|}{ SVAR $\left(U_{M}, i p, U_{F}\right)^{\prime}$} & \multicolumn{3}{|c|}{$\overline{\mathrm{SVAR}}\left(E P U, i p, U_{F}\right)^{\prime}$} \\
\hline & \multicolumn{3}{|c|}{ Fraction variation in $U_{M}$} & Fractio & variation $\mathrm{i}$ & EPU \\
\hline$s$ & $U_{M}$ Shock & ip Shock & $U_{F}$ Shock & EPU Shock & ip Shock & $U_{F}$ Shock \\
\hline 1 & {$[0.19,0.41]$} & {$[0.52,0.72]$} & {$[0.05,0.22]$} & {$[0.15,0.76]$} & {$[0.03,0.61]$} & {$[0.20,0.44]$} \\
\hline 12 & {$[0.22,0.46]$} & {$[0.39,0.57]$} & {$[0.13,0.36]$} & {$[0.10,0.46]$} & {$[0.02,0.34]$} & {$[0.52,0.70]$} \\
\hline$\infty$ & {$[0.25,0.49]$} & {$[0.39,0.59]$} & {$[0.10,0.30]$} & {$[0.09,0.38]$} & {$[0.02,0.28]$} & {$[0.60,0.75]$} \\
\hline \multirow[t]{2}{*}{$s_{\max }$} & {$[0.25,0.49]$} & {$[0.52,0.72]$} & {$[0.13,0.36]$} & {$[0.18,0.81]$} & {$[0.03,0.64]$} & {$[0.60,0.75]$} \\
\hline & \multicolumn{3}{|c|}{ Fraction variation in $i p$} & \multicolumn{3}{|c|}{ Fraction variation in $i p$} \\
\hline$s$ & $U_{M}$ Shock & ip Shock & $U_{F}$ Shock & EPU Shock & ip Shock & $U_{F}$ Shock \\
\hline 1 & {$[0.36,0.60]$} & {$[0.31,0.53]$} & {$[0.06,0.12]$} & {$[0.04,0.72]$} & {$[0.16,0.82]$} & {$[0.09,0.17]$} \\
\hline 12 & {$[0.07,0.21]$} & {$[0.40,0.60]$} & {$[0.31,0.40]$} & {$[0.03,0.58]$} & {$[0.12,0.64]$} & {$[0.26,0.37]$} \\
\hline$\infty$ & {$[0.02,0.07]$} & {$[0.47,0.64]$} & {$[0.32,0.51]$} & {$[0.13,0.74]$} & {$[0.04,0.60]$} & {$[0.19,0.29]$} \\
\hline \multirow[t]{2}{*}{$s_{\max }$} & {$[0.37,0.62]$} & {$[0.47,0.64]$} & {$[0.38,0.54]$} & {$[0.13,0.74]$} & {$[0.22,0.83]$} & {$[0.27,0.39]$} \\
\hline & \multicolumn{3}{|c|}{ Fraction variation in $U_{F}$} & \multicolumn{3}{|c|}{ Fraction variation in $U_{F}$} \\
\hline$s$ & $U_{M}$ Shock & ip Shock & $U_{F}$ Shock & EPU Shock & ip Shock & $U_{F}$ Shock \\
\hline 1 & {$[0.03,0.14]$} & {$[0.01,0.07]$} & {$[0.84,0.94]$} & {$[0.01,0.11]$} & {$[0.07,0.35]$} & {$[0.62,0.84]$} \\
\hline 12 & {$[0.08,0.23]$} & {$[0.00,0.05]$} & {$[0.76,0.89]$} & {$[0.02,0.30]$} & {$[0.06,0.48]$} & {$[0.41,0.66]$} \\
\hline$\infty$ & {$[0.11,0.28]$} & {$[0.02,0.05]$} & {$[0.70,0.86]$} & {$[0.04,0.38]$} & {$[0.06,0.51]$} & {$[0.35,0.59]$} \\
\hline$s_{\max }$ & {$[0.11,0.28]$} & {$[0.02,0.10]$} & {$[0.84,0.94]$} & {$[0.04,0.38]$} & {$[0.11,0.51]$} & {$[0.64,0.84]$} \\
\hline
\end{tabular}

Each panel shows the fraction of $s$-step-ahead forecast-error variance of the variable given in the panel title that is explained by the shock named in the column heading. The row denoted " $s=s_{\text {max }}$ " reports the maximum fraction of forecast error variance explained across all VAR forecast horizons s. The numbers in brackets represent the ranges for these numbers across all solutions in the identified set. The data are monthly and span the period 1960:07 to 2015:04. 


\section{Online Appendix}

\section{Sampling Simulation}

In point-identified models, sampling uncertainty can be evaluated using frequentist confidence intervals or Bayesian credible regions, and they coincide asymptotically. Inference for setidentified SVARs is, however, more challenging because no consistent point estimate is available. As pointed out in Moon and Schorfheide (2012), the credible regions of Bayesian identified impulses responses will be distinctly different from the frequentist confidence sets, with the implication that Bayesian error bands cannot be interpreted as approximate frequentist error bands. Our analysis is frequentist, and while the two applications presented above illustrate how the dynamic responses vary across estimated models, where each model is evaluated at a solution in $\overline{\mathcal{B}}(\mathbf{B} ; \overline{\mathbf{k}}, \overline{\boldsymbol{\tau}}, \mathbf{S})$, we still need a way to assess the robustness of our procedure, especially since it is new to the literature.

Unfortunately, few methods are available to evaluate the sampling uncertainty of set identified SVARs from a frequentist perspective, and these tend to be specific to the imposition of particular identifying restrictions. Moon, Schorfheide, and Granziera (2013) suggest a projections based method within a moment-inequality setup, but it is designed to study SVARs that only impose restrictions on one set of impulse response functions. Furthermore, the method is computationally intense, requiring a simulation of critical value for each rotation matrix. Gafarov, Meier, and Olea (2015) suggest to collect parameters of the reduced form model in a $1-\alpha$ Wald ellipsoid but the approach is conservative. For the method to get an exact coverage of $1-\alpha$, the radius of the Wald-ellipsoid needs to be carefully calibrated. As discussed in Kilian and Lutkepohl (2016), even with these adjustments, existing frequentist confidence sets for set-identified models still tend to be too wide to be informative. It is fair to say that there exists no generally agreed upon method for conducting inference in set-identified SVARs.

We use a bootstrap/Monte Carlo procedure to assess the sampling error of our inequality restrictions when $S_{t}$ and $G_{t}$ are variables external to the three variable SVAR.

Let $R$ be the number of replications in a repeated sampling experiment. Let "hats" denote estimated values from historical data, e.g., $\widehat{\mathbf{e}}_{t}$ denotes estimated structural shocks and $\widehat{\mathbf{B}}$ estimated structural covariance matrix. To denote simulated data, we use a "**, while to denote estimated values from simulated data, a "hat" is combined with a "*". To generate samples of the structural shocks from this solution in a way that ensures the events that appear in historical data also occur in our simulated samples, we draw randomly with replacement from the sample estimates of the shocks, $\widehat{\mathbf{e}}_{t}$, with the exception that we fix the values for these shocks in each replication in the periods $\bar{\tau}_{1} \bar{\tau}_{2}, \bar{\tau}_{3}, \bar{\tau}_{4}$ and $\bar{\tau}_{5}$, where $\bar{\tau}_{1}$ is the period 1987:10 of the stock market crash, $\bar{\tau}_{2}$ is 1970:12, $\bar{\tau}_{3} \in[2007: 12,2009: 06], \bar{\tau}_{4}$ is 1979:10 and $\bar{\tau}_{5} \in$ [2011:07, 2011:08]. Since we identify a set of estimated parameters $\widehat{\mathbf{B}}$ and therefore a set of estimated shocks $\widehat{\mathbf{e}}_{t}$, 
we generate $R$ samples of data from each $\widehat{\mathbf{e}}_{t}$ in the set. This is then repeated for every solution/shock sequence in the identified set to obtain a confidence region for the identified set of impulse responses.

Let $M$ be the number of solutions in the identified set $\overline{\mathcal{B}}(\mathbf{B} ; \overline{\mathbf{k}}, \overline{\boldsymbol{\tau}}, \mathbf{S})$ and let $m$ index an arbitrary solution in the set. Index each draw from the estimated shocks with $r$ and denote the $r$ th draw from the $m$ th solution as $\mathbf{e}_{t}^{m r}$. Each $\mathbf{e}_{t}^{m r}$ is combined with the $\mathbf{B}$ parameters of the $m$ th solution, $\widehat{\mathbf{B}}^{m}$ to generate $R$ samples of size $T$ of $\boldsymbol{\eta}_{t}^{m r *}=\widehat{\mathbf{B}}^{m} \mathbf{e}_{t}^{m r}$. Next, $R$ new samples of $\mathbf{X}_{t}$ are recursively generated for each replication $r=1, \ldots, R$ using $\mathbf{X}_{t}=\sum_{j=1}^{p} \widehat{\mathbf{A}}_{j} \mathbf{X}_{t-j}+\boldsymbol{\eta}_{t}^{m r *}$, with initial conditions fixed at their sample values, $\left[\mathbf{X}_{-p+1}, \ldots, \mathbf{X}_{0}\right]$. Using each of these new samples of $\mathbf{X}_{t}$, we fit a $\operatorname{VAR}(p)$ model to obtain new least squares estimates $\left[\widehat{\boldsymbol{\eta}}_{t}^{m r *}, \widehat{\mathbf{A}}_{1}^{m r *}, \ldots, \widehat{\mathbf{A}}_{p}^{m r *}\right]$ and $\widehat{\boldsymbol{\Omega}}^{m r *}=\operatorname{cov}\left(\widehat{\boldsymbol{\eta}}_{t}^{m r *}, \widehat{\boldsymbol{\eta}}_{t}^{m r *}\right)$, and $\hat{\mathcal{B}}^{m r *}=\left\{\widehat{\mathbf{B}}^{m r *}=\hat{\mathbf{P}}^{m r *} \mathbf{Q}: \mathbf{Q} \in \mathbb{O}_{n}, \operatorname{diag}\left(\widehat{\mathbf{B}}^{m r *}\right) \geq 0, \bar{g}_{Z}(\mathbf{B})=\mathbf{0}\right\}$, where where $\mathbb{O}_{n}$ is the set of $n \times n$ orthonormal matrices and $\hat{\mathbf{P}}^{m r *}$ is the unique lower triangular Cholesky factor of $\widehat{\Omega}^{m r *}$.

To generate samples of the external variables $S_{1 t}$ and $S_{2 t}$ from $m$ th solution in a way that ensures that the correlations with the uncertainty shocks that appear in our historical data also appear in our simulated samples, we first generate historical idiosyncratic stock market shocks $e_{S_{1} t}^{m}$ and gold price shocks $e_{S_{2} t}^{m}$ as the fitted residuals from regressions of $S_{1 t}$ and $S_{2 t}$ on a single autoregressive lag and on $\widehat{\mathbf{e}}_{t}$, respectively. Next, we draw randomly with replacement from $e_{S_{1} t}^{m}$ and $e_{S_{2} t}^{m}$ with the exception that, as above, we fix the values for these shocks in each replication in the periods $\bar{\tau}_{1}$ and $\bar{\tau}_{2}$, to obtain $r=1, \ldots, R$ new values $e_{S_{1} t}^{m r}$ and $e_{S_{2} t}^{m r}$ and $R$ new values of $S_{1 t}$ and $S_{2 t}$ by recursively iterating on

$$
\begin{aligned}
& S_{1 t}^{m r}=d_{01}^{m}+\widehat{\rho}_{1} S_{1 t-1}+\mathbf{d}_{1}^{m \prime} \mathbf{e}_{t}^{m r}+e_{S_{1} t}^{m r} \\
& S_{2 t}^{m r}=d_{02}^{m}+\widehat{\rho}_{1} S_{2 t-1}+\mathbf{d}_{2}^{m \prime} \mathbf{e}_{t}^{m r}+e_{S_{2} t}^{m r}
\end{aligned}
$$

with initial conditions fixed at their initial sample values, $\left[S_{11}, S_{21}\right]$. The parameters $\widehat{\rho}_{1}$ and $\widehat{\rho}_{2}$ are estimated from a first order autoregression for each variable. The parameters $\mathbf{d}_{1}^{m \prime}$ and $\mathbf{d}_{2}^{m \prime}$ in (A1) and (A2) are calibrated to target the observed correlations $\operatorname{corr}\left(S_{1 t}, \widehat{\mathbf{e}}_{t}^{m}\right)$ and $\operatorname{corr}\left(S_{2 t}, \widehat{\mathbf{e}}_{t}^{m}\right)$ for the $m$ th solution in historical data so that $\operatorname{corr}\left(S_{1 t}^{m r}, \mathbf{e}_{t}^{m r}\right)$ and $\operatorname{corr}\left(S_{2 t}^{m r}, \mathbf{e}_{t}^{m r}\right)$ roughly equal $\operatorname{corr}\left(S_{1 t}, \widehat{\mathbf{e}}_{t}^{m}\right)$ and $\operatorname{corr}\left(S_{2 t}, \widehat{\mathbf{e}}_{t}^{m}\right)$ on average across all replications $R$.

We construct confidence sets for the set of IRFs in repeated samples as follows. The number of replications is set to $R=1,000$. In each replication of each solution, $K=1.5$ million rotation matrices $\mathbf{Q}$ are entertained, but only $K_{m r} \leq K$ rotations will generate solutions that are admitted into the identified set for that replication, $\overline{\mathcal{B}}^{m r *}(\cdot)$. Let $\Theta_{i, j, s}^{m, r, k}$ be the $s$-period ahead response of the $i$ th variable to a standard deviation change in shock $j$ at the $k$-th rotation of $K_{m r}$, for 
replication $r$ and solution $m .^{20}$ Let $\underline{\Theta}_{i, j, s}^{m, r}=\min _{k \in\left[1, K_{m r}\right]} \Theta_{i, j, s}^{m, r, k}$ and $\bar{\Theta}_{i, j, s}^{m, r}=\max _{k \in\left[1, K_{m r}\right]} \Theta_{i, j, s}^{m, r, k}$. Each $\left(\underline{\Theta}_{i, j, s}^{m, r}, \bar{\Theta}_{i, j, s}^{m, r}\right)$ pair represents the extreme (highest and lowest) dynamic responses in replication $r$ of solution $m$. From the quantiles of the set $\left\{\underline{\Theta}_{i, j, s}^{m, r}\right\}_{m=1, r=1}^{M, R}$ that includes all replications for all solutions we can obtain the $\alpha / 2$ critical point $\underline{\Theta}_{i, j, s}(\alpha / 2)$. Similarly, from the quantiles of $\left\{\bar{\Theta}_{i, j, s}^{m, r}\right\}_{m=1, r=1}^{M, R}$, we have the $1-\alpha / 2$ critical point $\bar{\Theta}_{i, j, s}(1-\alpha / 2)$. Eliminating the lowest and highest $\alpha / 2$ percent of the samples gives a $(1-\alpha) \%$ percentile-based confidence interval defined by

$$
C I_{\alpha, g}=\left[\underline{\Theta}_{i, j, s}(\alpha / 2), \bar{\Theta}_{i, j, s}(1-\alpha / 2)\right] .
$$

$C I_{\alpha, g}$ denotes the confidence intervals for sets of solutions that satisfy all constraints, including the event and correlation constraints: $\bar{g}_{Z}(\mathbf{B})=0, \bar{g}_{E}(\mathbf{B} ; \bar{\tau}, \overline{\mathbf{k}}) \geq 0, \bar{g}_{C}(\mathbf{B} ; \mathbf{S}) \geq 0$. We use $C I_{\alpha, g_{Z}}$ to denote the confidence intervals for sets of solutions that satisfy only the reduced form covariance restrictions $\bar{g}_{Z}(\mathbf{B})=0$.

${ }^{20}$ The $s$-period ahead dynamic responses to one-standard deviation shocks in the $j /$ th variable are defined as

$$
\frac{\partial \mathbf{X}_{t+s}}{\partial e_{j t}}=\widehat{\mathbf{\Psi}}_{s}^{m r *} \widehat{\mathbf{b}}^{m r k j *}
$$

where $\widehat{\mathbf{b}}^{m r k j *}$ is the $j$ th column of $\widehat{\mathbf{B}}^{m r k *}$ and the coefficient matrixes $\widehat{\boldsymbol{\Psi}}_{s}^{m r *}$ are given by $\widehat{\boldsymbol{\Psi}}^{m r *}(L)=\widehat{\boldsymbol{\Psi}}_{0}^{m r *}+$ $\widehat{\mathbf{\Psi}}_{1}^{m r *} L+\widehat{\mathbf{\Psi}}_{2}^{m r *} L^{2}+\ldots=\widehat{\mathbf{A}}^{m r *}(L)^{-1}$. 